\title{
Theoretical Performance Limits of Massive MIMO with Uncorrelated Rician Fading Channels
}

\author{
Luca Sanguinetti, Senior Member, IEEE, Abla Kammoun, Member, IEEE, and Merouane Debbah, Fellow, IEEE
}

\begin{abstract}
This work considers a multicell Massive MIMO network with $L$ cells, each comprising a BS with $M$ antennas and $K$ single-antenna user equipments. Within this setting, we are interested in deriving approximations of the achievable rates in the uplink and downlink under the assumption that single-cell linear processing is used at each BS and that each intracell link forms an uncorrelated MIMO Rician fading channel matrix; that is, with a deterministic line-of-sight (LoS) path and a stochastic non-line-of-sight component describing a spatial uncorrelated multipath environment. The analysis is conducted assuming that $N$ and $K$ grow large with a given ratio $N / K$ under the assumption that the data transmission in each cell is affected by channel estimation errors, pilot contamination, an arbitrary large scale attenuation and LoS components. Numerical results are used to prove that the approximations are asymptotically tight, but accurate for systems with finite dimensions under different operating conditions. The asymptotic results are also used to evaluate the impact of LoS components. In particular, we exemplify how the number of antennas for achieving a target rate can be substantially reduced with LoS links of only a few dBs of strength.
\end{abstract}

Index Terms-Massive MIMO, uncorrelated Rician fading channels, asymptotic analysis, random matrix theory, noncentered random channel estimates, pilot contamination.

\section{INTRODUCTION}

Massive MIMO refers to a wireless network technology where base stations (BSs) are equipped with a very large number $M$ of antennas to serve a multitude of user equipments (UEs) by spatial multiplexing [1], [2]. Exciting developments have occurred in the recent year. In industry, the technology has been integrated into the 5G New Radio standard [3]. In academia, the long-standing pilot contamination issue, which was believed to impose fundamental limitations [1], [4], has finally been resolved [5]. More precisely, [5] used optimal processing under correlated Rayleigh fading channel models [6. Sect. 2.4] and proved that an unbounded capacity (as $M \rightarrow \infty$ ) is achieved with Massive MIMO when the channel covariance matrices of the pilot contaminating UEs are asymptotically linearly independent, which is generally the case in practice.

In this work, we consider both uplink (UL) and downlink (DL) of a Massive MIMO network with $L$ cells, each comprising a BS with $M$ antennas and $K$ single-antenna

L. Sanguinetti is with the University of Pisa, Dipartimento di Ingegneria dell'Informazione, 56122, Italy (luca.sanguinetti@unipi.it). A. Kammoun is with the Electrical Engineering Department, King Abdullah University of Science and Technology, Thuwal, Saudi Arabia (abla.kammoun@gmail.com). M. Debbah is with the Mathematical and Algorithmic Sciences Lab, Huawei Technologies Co. Ltd., France (merouane.debbah@huawei.com).

A preliminary version of this paper was presented at IEEE ICASSP'17, New Orleans, USA, March 2017.

This work was partially supported by the University of Pisa under the PRA 2018-2019 Research Project CONCEPT and also by the H2020-ERC PoCCacheMire project (grant 727682)
UEs. We assume that the system is affected by channel estimation errors, pilot contamination, and an arbitrary large scale attenuation. Single-cell linear processing is used at the BSs [6, Sect. 4.1.1]. In particular, we assume that in the DL maximum ratio transmit (MRT) or regularized zero forcing (RZF) are used as precoding techniques, whereas maximum ratio combining (MRC) or minimum mean square error (MMSE) combing are used in the UL for data recovery. Inspired by [7], we aim at deriving approximations of the achievable rates. The analysis is conducted assuming that $N$ and $K$ grow large with a non-trivial ratio $N / K$. Unlike [7] and most of the existing literature on the asymptotic analysis of Massive MIMO systems, we model the intracell communication links as uncorrelated Rician fading, which is more general and accurate to capture the fading variations when there is a lineof-sight (LoS) component.

\section{A. Contributions}

Compared to Rayleigh fading, a Rician channel model makes the asymptotic analysis of Massive MIMO much more involved than in [7]. To overcome this issue, recent results from random matrix theory and large system analysis [8], [9] are used to compute asymptotic expressions of the signal-tointerference-plus-noise ratios (SINRs), which are eventually used to approximate the achievable rates. The approximations are proven to be asymptotically tight, but accurate for realistic system dimensions, by means of the numerical results. As a notable outcome of this work, the above asymptotic analysis provides an analytical framework that can be used to evaluate the network performance under different settings without resorting to heavy Monte Carlo simulations. Also, it can be used to eventually get insights into how the LoS components in each cell affect channel estimation errors, intercell interference, and pilot contamination. To exemplify this, we consider a simplified channel model wherein the LoS vectors of different UEs are mutually orthogonal and analyze to which extent the presence of LoS components, with only a few dBs of strength, bring potential benefits to the SE of the network. To further quantify this, we numerically compute the number of antennas needed to achieve a given average rate with Rician fading and show this is substantially smaller than with Rayleigh fading. This confirms that if the UEs are scheduled properly (such that the interference between their LoS components vanish as the number of antennas grows) Rician fading may be beneficial for Massive MIMO [10]. The conference version [11] of this paper contains only a subset of the analysis above (and has no technical proofs). 


\section{B. Main literature}

The main literature related to this work is represented by [7], [12]-[23]. Tools from random matrix theory are used in [12] to compute the ergodic sum rate in a single-cell MIMO setting with Rayleigh fading and different precoding schemes while the multicell case is analyzed in [13]. Similar tools are used in [14] to solve the power minimization problem under different configurations of cooperation among BSs. A similar large system analysis is presented in [7] for the UL and DL of Massive MIMO in cellular networks, wherein channel estimation and pilot contamination are also taken into account. All these works relies on random matrix theory tools but assume Rayleigh fading channels. With Rician fading channels, the asymptotic analysis is much more demanding with the main difficulty lying in the correlation induced by pilot contamination among the non-centered random channel estimates. In [16], the authors investigate a LoS-based conjugate beamforming transmission scheme and derive some expressions of the statistical SINR under the assumption that $N$ grows large and $K$ is fixed. In [17], the authors study the fluctuations of the mutual information of a cooperative small cell network operating over a Rician fading channel under the form of a central limit theorem and provide an explicit expression of the asymptotic variance. In [18], a deterministic equivalent of the ergodic sum rate and an algorithm for evaluating the capacity achieving input covariance matrices for the UL of a large-scale MIMO are proposed for spatially correlated MIMO channel with LoS components. In [19], the authors derive tractable expressions for the achievable UL rate for $\mathrm{ZF}$ and $\mathrm{MRC}$ in the large-antenna limit, along with approximating results that hold for any finite number of antennas ( $N$ grows large and $K$ is fixed). Based on these analytical results, the transmit power scaling law to meet a desirable quality of service is computed. A numerical analysis is used in [20] to show how LoS components may potentially improve the system performance and mitigate the pilot contamination problem. In [21], a full-duplex multicell Massive MIMO systems is analyzed. A deterministic approximation of the UL achievable rate with $\mathrm{MRC}$ is derived based on random matrix theory. It is then proved that the BS-to-BS interference and self interference asymptotically vanishes. In [24], the authors study the ergodic secrecy sum rate in the DL of a multiuser MIMO system with RZF. Unlike this work where CSI is acquired by using an UL pilot training phase that induces pilot contamination, the imperfect CSI is modelled by using the generic Gauss-Markov formulation. In [22], a detailed achievable rate analysis of regular and large-scale singleuser MIMO systems is presented under transceiver hardware impairments and Rician fading conditions. In [25], [26], the UL SE of Massive MIMO communications with analogdigital transceivers over Rician fading channels is investigated. Unlike this work, only MRC with perfect and imperfect CSI is considered, and the asymptotic analysis is carried out in the regime where $N$ grows large and $K$ is fixed. In [27], spatially correlated Rician fading channels are considered and the closed-form expressions for the SE with both MRC and MRT are derived with different channel estimation schemes.

\section{Organization and notation}

The remainder of this paper is organized as follows. Next section describes the system model and derives achievable rates for UL and DL with single-cell linear receive combining and transmit precoding. Section III contains our main technical results wherein we derive asymptotically tight approximations for UL and DL achievable rates. A simplified channel model is also considered to get instrumental insights into the impact of LoS components on the network performance. In Section $\mathrm{V}$ the asymptotic analysis is numerically validated. Finally, the major conclusions and implications are drawn in Section VI All the technical proofs are presented in the Appendices.

The following notation is used throughout the paper. Scalars are denoted by lower case letters whereas boldface lower (upper) case letters are used for vectors (matrices). We denote by $\mathbf{I}_{N}$ the identity matrix of order $N$ and call $[\mathbf{A}]_{i, k}$ the $(i, k)$ th element of $\mathbf{A}$. A random vector $\mathbf{x} \sim \mathcal{C N}(\mathbf{m}, \mathbf{C})$ is complex Gaussian distributed with mean $\mathbf{m}$ and covariance matrix C. The trace, transpose, conjugate transpose, real part, and expectation operators are denoted by $\operatorname{tr}(\cdot),(\cdot)^{T},(\cdot)^{H}, \operatorname{Re}\{\cdot\}$, and $\mathbb{E}\{\cdot\}$. We use $\mathbb{E}\{\cdot \mid \cdot\}$ to denote the conditional expectation operator. We use $a_{n} \asymp b_{n}$ to denote $a_{n}-b_{n} \rightarrow_{n \rightarrow \infty} 0$ (almost surely) for two (random) sequences $a_{n}, b_{n}$.

\section{SYSTEM MODEL}

Consider a Massive MIMO system composed of $L$ cells, the BS of each cell is equipped with $N$ antennas and communicates with $K$ single-antenna UEs. A double index notation is used to refer to each UE as e.g., "user $k$ in cell $j$ ". Under this convention, let $\mathbf{h}_{j l k} \in \mathbb{C}^{N}$ be the channel from UE $k$ in cell $l$ to BS $j$ within a coherence block 1 We model the channel vectors $\left\{\mathbf{h}_{j j k} ; k=1, \ldots, K\right\}$, i.e. from the $K$ UEs in cell $j$ to $\mathrm{BS} j$, as uncorrelated Rician fading. On the other hand, an uncorrelated Rayleigh fading model is assumed for $\left\{\mathbf{h}_{j l k} ; k=1, \ldots, K\right\}$ with $l \neq j$. This choice is motivated by the fact that the achievable rates under this model are close to those under practical measured channels with LoS and spatially distributed UEs [28]. Under the above model, we have that $\mathbf{h}_{j l k}=\sqrt{\beta_{j l k}} \mathbf{w}_{j l k}$ where $\beta_{j l k}$ accounts for the corresponding large scale channel fading or pathloss (from BS $j$ to UE $k$ in cell $l$ ) and $\mathbf{w}_{j l k} \in \mathbb{C}^{N}$ is the small scale fading channel. The channel matrix $\mathbf{H}_{j l} \in \mathbb{C}^{N \times K}$ from cell $l$ to BS $j$ is thus given by $\mathbf{H}_{j l}=\left[\mathbf{h}_{j l 1}, \ldots, \mathbf{h}_{j l K}\right]$. We model $\mathbf{w}_{j l k}$ as

$$
\mathbf{w}_{j l k}=\left\{\begin{array}{cc}
\sqrt{\frac{1}{1+\kappa_{j k}}} \mathbf{z}_{j j k}+\sqrt{\frac{\kappa_{j k}}{1+\kappa_{j k}}} \mathbf{a}_{j j k} & l=j \\
\mathbf{z}_{j l k} & l \neq j
\end{array}\right.
$$

where $\mathbf{z}_{j l k} \in \mathbb{C}^{N} \sim \mathcal{C N}\left(\mathbf{0}_{N}, \mathbf{I}_{N}\right), \mathbf{a}_{j j k} \in \mathbb{C}^{N}$ is a deterministic vector that accounts for the LoS component, and the scalar $\kappa_{j k} \geq 0$ is the Rician factor denoting the power ratio between $\mathbf{a}_{j j k}$ and $\mathbf{z}_{j j k}$. For notational convenience, we let

$$
d_{j l k}=\left\{\begin{array}{cc}
\frac{\beta_{j j k}}{1+\kappa_{j k}} & l=j \\
\beta_{j l k} & l \neq j
\end{array}\right.
$$

\footnotetext{
${ }^{1} \mathrm{~A}$ coherence block consists of a number of subcarriers and time samples over which the channel response can be approximated as constant and flatfading (e.g. [6 Sect. 2.1])
} 
such that $\mathbf{h}_{j l k}$ can be rewritten as

$$
\mathbf{h}_{j l k}=\left\{\begin{array}{cc}
\widetilde{\mathbf{h}}_{j j k}+\overline{\mathbf{h}}_{j j k} & l=j \\
\widetilde{\mathbf{h}}_{j l k} & l \neq j
\end{array}\right.
$$

with $\widetilde{\mathbf{h}}_{j l k}=\sqrt{d_{j l k}} \mathbf{z}_{j l k}$ and $\overline{\mathbf{h}}_{j j k}=\sqrt{d_{j j k} \kappa_{j k}} \mathbf{a}_{j j k}$. While $\widetilde{\mathbf{h}}_{j j k}$ accounts for the small-scale fading variations of $\mathbf{h}_{j j k}$, $\overline{\mathbf{h}}_{j j k}$ depends on the large-scale fading components of propagation channel and evolves slowly in time compared to $\widetilde{\mathbf{h}}_{j j k}$. Measurements in [29] suggest roughly two orders of magnitude slower variations. In practice, this means that $\overline{\mathbf{h}}_{j j k}$ maintains constant for a sufficiently large number of reception phases to be accurately estimated at the BS. Therefore, in the subsequent analysis we assume that $\overline{\mathbf{h}}_{j j k}$ is perfectly known, which is the common practice in communication theory.

\section{A. Channel estimation}

Pilot-based channel training is utilized to estimate the channel matrix $\mathbf{H}_{j j}$ at $\mathrm{BS} j$. We assume that the BS and UEs are perfectly synchronized and operate according to a time-division duplex (TDD) protocol wherein the DL data transmission phase is preceded in the UL by a training phase for channel estimation [1], [10], [30]. In Massive MIMO, it is reasonable to expect that the number of UEs per cell will be very large. Due to the limited number of orthogonal pilot sequences, the same set of orthogonal pilot sequences is utilized for channel estimation in each cell (i.e., the pilot reuse factor is one). This results into pilot contamination in the channel estimation [1], [4]-[6]. If an MMSE estimator is used [7], then the estimate $\widehat{\mathbf{h}}_{j l k}$ of $\mathbf{h}_{j l k} \forall j, k$ is given by [31]

$$
\widehat{\mathbf{h}}_{j l k}=\left\{\begin{array}{cc}
\overline{\mathbf{h}}_{j j k}+\frac{d_{j j k}}{\frac{1}{\rho^{\mathrm{tr}}}+\sum_{n=1}^{L} d_{j n k}}\left(\mathbf{y}_{j k}^{\mathrm{tr}}-\overline{\mathbf{h}}_{j j k}\right) & l=j \\
\frac{d_{j l k}}{\frac{1}{\rho^{\mathrm{tr}}}+\sum_{n=1}^{L} d_{j n k}}\left(\mathbf{y}_{j k}^{\mathrm{tr}}-\overline{\mathbf{h}}_{j j k}\right) & l \neq j
\end{array}\right.
$$

where $\rho^{\text {tr }}$ accounts for the SNR during the UL training phase and $\mathbf{y}_{j k}^{\mathrm{tr}}$ is given by

$$
\mathbf{y}_{j k}^{\mathrm{tr}}=\mathbf{h}_{j j k}+\sum_{l=1, l \neq j}^{L} \mathbf{h}_{j l k}+\frac{1}{\sqrt{\rho^{\mathrm{tr}}}} \mathbf{n}_{j k}^{\mathrm{tr}}
$$

with $\mathbf{n}_{j k}^{\mathrm{tr}} \sim \mathcal{C N}\left(\mathbf{0}_{N}, \mathbf{I}_{N}\right)$. The estimate $\widehat{\mathbf{h}}_{j j k}$ is distributed as $\widehat{\mathbf{h}}_{j j k} \sim \mathcal{C N}\left(\overline{\mathbf{h}}_{j j k}, \phi_{j j k} \mathbf{I}_{N}\right)$ with

$$
\phi_{j l k}=\frac{d_{j j k} d_{j l k}}{\frac{1}{\rho^{\mathrm{tr}}}+\sum_{n=1}^{L} d_{j n k}} .
$$

The estimated UL channel of cell $j$ is thus given by $\widehat{\mathbf{H}}_{j j}=$ $\left[\widehat{\mathbf{h}}_{j j 1}, \ldots, \widehat{\mathbf{h}}_{j j K}\right]$. According to the orthogonality principle [31], the estimation error $\mathbf{e}_{j j k}=\mathbf{h}_{j j k}-\widehat{\mathbf{h}}_{j j k}$ is independent from $\widehat{\mathbf{h}}_{j j k}$ and distributed as $\sim \mathcal{C N}\left(\mathbf{0}_{N},\left(d_{j j k}-\phi_{j j k}\right) \mathbf{I}_{N}\right)$.

\section{B. Uplink}

Denoting by $\mathbf{v}_{j k} \in \mathbb{C}^{N}$ the receiving combiner of UE $k$ in cell $j$, its received signal is given by

$$
y_{j k}^{\mathrm{ul}}=\sum_{i=1}^{K} \mathbf{v}_{j k}^{H} \mathbf{h}_{j j i} s_{j i}^{\mathrm{ul}}+\sum_{l=1, l \neq j}^{L} \sum_{i=1}^{K} \mathbf{v}_{j k}^{H} \mathbf{h}_{j l i} s_{l i}^{\mathrm{ul}}+\mathbf{v}_{j k}^{H} \mathbf{n}_{j k}^{\mathrm{ul}}
$$

where $s_{l i}^{\mathrm{ul}} \in \mathbb{C}$ is the signal transmitted in the UL from UE $i$ in cell $l$, assumed independent across $(l, i)$ pairs, of zero mean and unit variance, and $\mathbf{n}_{j k}^{\mathrm{ul}} \sim \mathcal{C} \mathcal{N}\left(\mathbf{0}, 1 / \rho^{\mathrm{ul}} \mathbf{I}_{N}\right)$ where $\rho^{\mathrm{ul}}$ accounts for the signal-to-noise ratio (SNR) in the UL. The SE that a UE can achieve is upper bounded by the channel capacity, thus an achievable SE is any number that is below the capacity. While the classical "Shannon formula" cannot be applied when the receiver has imperfect CSI, there are well-established capacity lower bounds that can be used. With MMSE channel estimation, a standard lower bound in Massive MIMO [4], [6], [7] allows to compute an achievable SE in the $\mathrm{UL}$ as $r_{j k}^{\mathrm{ul}}=\mathbb{E}_{\widehat{\mathbf{H}}_{j j}}\left\{\log _{2}\left(1+\gamma_{j k}^{\mathrm{ul}}\right)\right\}$ where $\mathbb{E}_{\widehat{\mathbf{H}}_{j j}}\{\cdot\}$ denotes the expectation with respect to $\widehat{\mathbf{H}}_{j j}$ and $\gamma_{j k}^{\mathrm{ul}}$ is the SINR given by (7) on the top of next page. As mentioned earlier, we consider MRC and single-cell MMSE (S-MMSE) as detection schemes. Then, the combiner vector $\mathbf{v}_{j k}$ is given by [6

$$
\begin{aligned}
\mathbf{v}_{j k}^{\mathrm{MRC}} & =\widehat{\mathbf{h}}_{j j k} \\
\mathbf{v}_{j k}^{\mathrm{S}-\mathrm{MMSE}} & =\left(\sum_{i=1}^{K} \widehat{\mathbf{h}}_{j j i} \widehat{\mathbf{h}}_{j j i}^{H}+\left(\xi_{j}+N \varphi_{j}^{\mathrm{ul}}\right) \mathbf{I}_{N}\right)^{-1} \widehat{\mathbf{h}}_{j j k}
\end{aligned}
$$

where $\varphi_{j}^{\mathrm{ul}}>0$ is a design parameter and

$$
\xi_{j}=\sum_{l=1, l \neq j}^{L} \sum_{i=1}^{K} d_{j l i}+\sum_{i=1}^{K}\left(d_{j j i}-\phi_{j j i}\right) .
$$

The "single-cell" notion in (10) is used to differentiate it from the multicell MMSE (M-MMSE) combining scheme considered in [32], [33] for uncorrelated Rayleigh fading channels and in [5], [34] for correlated ones. The main difference from M-MMSE is that only the channel estimates $\widehat{\mathbf{H}}_{j j}$ in the own cell are computed and used in S-MMSE [5], [6]. The computational complexity of S-MMSE is thus lower than with M-MMSE, though the pilot overhead is identical since the same pilots can be used to estimate both intracell and inter-cell channels. However, notice that M-MMSE is optimal [5], [34] and provides unbounded capacity (in the regime $M \rightarrow \infty$ and $K$ kept fixed) for correlated Rayleigh fading channels [5]. In the case of uncorrelated channels (i.e., no spatial correlation), M-MMSE combining achieves only marginal gains compared to S-MMSE and is also fundamentally limited by pilot contamination as all other 'suboptimal' single-cell processing schemes [5], [34]. Since uncorrelated channels are considered in this work, we limit to consider SMMSE to make the problem analytically more tractable. The asymptotic analysis of M-MMSE combining/precoding with spatially correlated Rician fading channels is interesting but left for future work since it requires more advanced random matrix theory tools. 


$$
\gamma_{j k}^{\mathrm{ul}}=\frac{\left|\mathbf{v}_{j k}^{H} \widehat{\mathbf{h}}_{j j k}\right|^{2}}{\mathbb{E}\left\{\mathbf{v}_{j k}^{H}\left(\sum_{l=1, l \neq j}^{L} \sum_{i=1}^{K} \mathbf{h}_{j l i} \mathbf{h}_{j l i}^{H}+\sum_{i=1, i \neq k}^{K} \mathbf{h}_{j j i} \mathbf{h}_{j j i}^{H}+\mathbf{e}_{j j k} \mathbf{e}_{j j k}^{H}+\frac{1}{\rho^{\mathrm{ul}}} \mathbf{I}_{N}\right) \mathbf{v}_{j k} \mid \widehat{\mathbf{H}}_{j j}\right\}} .
$$

\section{Downlink}

Denoting by $\mathbf{g}_{j k} \in \mathbb{C}^{N}$ the precoding vector of UE $k$ in cell $j$, the received signal reads

$$
y_{j k}^{\mathrm{dl}}=\sum_{i=1}^{K} \mathbf{h}_{j j k}^{H} \mathbf{g}_{j i} s_{j i}^{\mathrm{dl}}+\sum_{l=1, l \neq j}^{L} \sum_{i=1}^{K} \mathbf{h}_{l j k}^{H} \mathbf{g}_{l i} s_{l i}^{\mathrm{dl}}+n_{j k}^{\mathrm{dl}}
$$

where $s_{l i}^{\mathrm{ul}} \in \mathbb{C}$ is the DL data symbol intended to UE $i$ in cell $l$, assumed independent across $(l, i)$ pairs, of zero mean and unit-variance, and $n_{j k}^{\mathrm{dl}} \sim \mathcal{C} \mathcal{N}\left(0,1 / \rho^{\mathrm{dl}}\right)$ where $\rho^{\mathrm{dl}}$ accounts for the signal-to-noise ratio (SNR) in the DL. As in [1], [7], [35], [36] (among many others), we assume that there are no downlink pilots such that the UEs do not have knowledge of the current channels but can only learn the average channel gain $\mathbb{E}\left\{\mathbf{h}_{j j k}^{H} \mathbf{g}_{j k}\right\}$. A well-established capacity lower bound that can be used within this setting is the useand-then-forget (UatF) bound [6, Sec. 4.3], whose name comes from the fact that channel estimates are used for designing the receive combining vectors and then effectively "forgotten" before signal detection. By applying the UatF bound, an achievable SE in the DL for UE $k$ in cell $j$ is obtained as $r_{j k}^{\mathrm{dl}}=\log _{2}\left(1+\gamma_{j k}\right)$ where $\gamma_{j k}^{\mathrm{dl}}$ is given by

$$
\gamma_{j k}^{\mathrm{dl}}=\frac{\left|\mathbb{E}\left\{\mathbf{h}_{j j k}^{H} \mathbf{g}_{j k}\right\}\right|^{2}}{\frac{1}{\rho_{\mathrm{dl}}}+\sum_{l=1}^{L} \sum_{i=1}^{K} \mathbb{E}\left\{\left|\mathbf{h}_{l j k}^{H} \mathbf{g}_{l i}\right|^{2}\right\}-\left|\mathbb{E}\left\{\mathbf{h}_{j j k}^{H} \mathbf{g}_{j k}\right\}\right|^{2}}
$$

where the expectation is taken with respect to the channel realizations. The above result holds true for any precoding scheme and is obtained by treating the inter-user interference (from the same and other cells) and channel uncertainty as worst-case Gaussian noise. As said earlier, we consider MRT and RZF as precoding schemes [1], [2], [7], [35]. This yields

$$
\begin{aligned}
& \mathbf{g}_{j k}^{\mathrm{MRT}}=\frac{\widehat{\mathbf{h}}_{j j k}}{\sqrt{\mathbb{E}\left\{\frac{1}{K} \sum_{k=1}^{K}\left\|\widehat{\mathbf{h}}_{j j k}\right\|^{2}\right\}}}=\sqrt{\theta_{j}} \widehat{\mathbf{h}}_{j j k} \\
& \mathbf{g}_{j k}^{\mathrm{RZF}}=\frac{\widehat{\mathbf{u}}_{j k}}{\sqrt{\mathbb{E}\left\{\frac{1}{K} \sum_{k=1}^{K}\left\|\widehat{\mathbf{u}}_{j k}\right\|^{2}\right\}}}=\sqrt{\psi_{j}} \widehat{\mathbf{u}}_{j k}
\end{aligned}
$$

where $\widehat{\mathbf{u}}_{j k}=\left(\sum_{i=1}^{K} \widehat{\mathbf{h}}_{j j i} \widehat{\mathbf{h}}_{j j i}^{H}+\left(\xi_{j}+N \varphi_{j}^{\mathrm{dl} l}\right) \mathbf{I}_{N}\right)^{-1} \widehat{\mathbf{h}}_{j j k}$ or, equivalently, $\widehat{\mathbf{u}}_{j k}=\frac{1}{N} \mathbf{Q}_{j} \widehat{\mathbf{h}}_{j j k}$ with

$$
\mathbf{Q}_{j}=\left(\frac{1}{N} \sum_{i=1}^{K} \widehat{\mathbf{h}}_{j j i} \widehat{\mathbf{h}}_{j j i}^{H}+\left(\frac{1}{N} \xi_{j}+\varphi_{j}^{\mathrm{dl}}\right) \mathbf{I}_{N}\right)^{-1}
$$

where $\varphi_{j}^{\mathrm{dl}} \geq 0$ is a design parameter.

\section{ASYMPTOTIC SPECTRAL EFFICIENCY}

We exploit the statistical distribution for the channels $\left\{\mathbf{H}_{j l}\right\}$ and the large dimensions of $N$ and $K$ to compute a deterministic approximation of $\gamma_{j k}$ in UL and DL, which will be eventually used to find an approximation of the ergodic sum rate. In doing so, we assume the following.

Assumption 1. $N$ and $K$ grow to infinity at the same pace, that is $1 \leq \liminf _{N} N / K \leq \lim \sup _{N} N / K<\infty$.

The above assumption will be referred to as $N, K \rightarrow \infty$ in the sequel. For technical reasons, the following reasonable assumption are is imposed [7]-[9], [12].

Assumption 2. As $N \rightarrow \infty$, we have that $\forall j, l, k$ $\lim \inf _{N} \beta_{j l k}>0$ and $\operatorname{lim\operatorname {sup}_{N}} \beta_{j l k}<\infty$, and also that $\forall j \lim \sup _{N}\left\|\overline{\mathbf{H}}_{j j}\right\|<\infty$.

The conditions on $\beta_{j l k}$ are a well established way to model that the array gathers more energy as $N$ increases [7], [12]. On the other hand, the condition on $\overline{\mathbf{H}}_{j j}$ implies that the Euclidean norm of the columns $\overline{\mathbf{h}}_{j j k}$ are uniformly bounded in $N, K[8]$, [9]. For simplicity, in the remainder we assume that $\varphi_{j}^{\mathrm{ul}}=$ $\varphi_{j}^{\mathrm{dl}}=\varphi_{j}$ and call $\lambda_{j}=\frac{1}{N} \xi_{j}+\varphi_{j}$ with

$$
\lambda_{j}=\frac{1}{N} \sum_{l=1, l \neq j}^{L} \sum_{i=1}^{K} d_{j l i}+\frac{1}{N} \sum_{i=1}^{K}\left(d_{j j i}-\phi_{j j i}\right)+\varphi_{j}
$$

such that $\mathbf{v}_{j k}^{\mathrm{S}-\mathrm{MMSE}}=\widehat{\mathbf{u}}_{j k}=\frac{1}{N} \mathbf{Q}_{j} \widehat{\mathbf{h}}_{j j k}$ and $\mathbf{g}_{j k}^{\mathrm{RZF}}=$ $\sqrt{\psi_{j}} \mathbf{v}_{j k}^{\mathrm{S}-\mathrm{MMSE}}$. Notice that (19) follows from (11).

\section{A. MRC and MRT}

Our first results are asymptotic approximations of the SINRs with MRC and MRT.

Lemma 1 (MRT). Let Assumptions 1 - 2 hold true. If MRT is employed, then $\gamma_{j k}^{\mathrm{MRT}} \asymp \bar{\gamma}_{j k}^{\mathrm{MRT}}$ with $\gamma_{j k}^{\mathrm{MRT}}$ given in (17) where $\bar{s}_{j k}$ takes the form

$$
\begin{aligned}
\bar{s}_{j k} & =\frac{1}{N} \sum_{l=1}^{L} \sum_{i=1}^{K} \bar{\theta}_{l} d_{l j k}\left(\phi_{l l i}+\frac{1}{N} \overline{\mathbf{h}}_{l l i}^{H} \overline{\mathbf{h}}_{l l i}\right) \\
& +\frac{1}{N} \sum_{i=1, i \neq k}^{K} \bar{\theta}_{j}\left(\phi_{j j i} \frac{1}{N} \overline{\mathbf{h}}_{j j k}^{H} \overline{\mathbf{h}}_{j j k}\right)
\end{aligned}
$$

and $\bar{\theta}_{l}$ is given by

$$
\bar{\theta}_{l}=\left(\frac{1}{K} \sum_{k=1}^{K}\left(\phi_{l l k}+\frac{1}{N} \overline{\mathbf{h}}_{l l k}^{H} \overline{\mathbf{h}}_{l l k}\right)\right)^{-1} .
$$

Proof: The proof is given in Appendix A using standard random matrix tools. Notice that this is not necessarily required for MRT since similar results can be obtained by computing the statistical expectations in (13) (e.g., [27]). 


$$
\begin{aligned}
& \bar{\gamma}_{j k}^{\mathrm{MRT}}=\frac{\bar{\theta}_{j}\left(\phi_{j j k}+\frac{1}{N} \overline{\mathbf{h}}_{j j k}^{H} \overline{\mathbf{h}}_{j j k}\right)^{2}}{\underbrace{\frac{1}{N \rho^{\mathrm{dl}}}}_{\text {Noise }}+\underbrace{\bar{s}_{j k}}_{\text {Non-coherent interference }}+\underbrace{\bar{\theta}_{j} \sum_{i=1, i \neq k}^{K}\left|\frac{1}{N} \overline{\mathbf{h}}_{j j i}^{H} \overline{\mathbf{h}}_{j j k}\right|^{2}+\sum_{l=1, l \neq j}^{L} \bar{\theta}_{l} \phi_{l j k}^{2}}_{\text {Coherent interference }}} \\
& \gamma_{j k}^{\mathrm{MRC}}=\frac{\left(\phi_{j j k}+\frac{1}{N} \overline{\mathbf{h}}_{j j k}^{H} \overline{\mathbf{h}}_{j j k}\right)^{2}}{\underbrace{\frac{1}{N \rho^{\mathrm{ul} \bar{\theta}_{j}}}}_{\text {Noise }}+\underbrace{\bar{s}_{j k}}_{\text {Non-coherent interference }}+\underbrace{\sum_{i=1, i \neq k}^{K}\left|\frac{1}{N} \overline{\mathbf{h}}_{j j k}^{H} \overline{\mathbf{h}}_{j j i}\right|^{2}+\sum_{l=1, l \neq j}^{L} \phi_{j l k}^{2}}_{\text {Coherent interference }}}
\end{aligned}
$$

Unlike the former approach, the latter does not apply to MRC. This is why random matrix tools are used in Appendix A.

Lemma 2 (MRC). Let Assumptions 1- 2 hold true. If MRC is employed, then $\gamma_{j k}^{\mathrm{MRC}} \asymp \bar{\gamma}_{j k}^{\mathrm{MRC}}$ with $\gamma_{j k}^{\mathrm{MRC}}$ given by 18) where $\bar{s}_{j k}$ is given by

$$
\begin{aligned}
\bar{s}_{j k} & =\frac{1}{N} \sum_{l=1}^{L} \sum_{i=1}^{K} d_{j l i}\left(\phi_{j j k}+\frac{1}{N} \overline{\mathbf{h}}_{j j k}^{H} \overline{\mathbf{h}}_{j j k}\right) \\
& +\frac{1}{N} \sum_{i=1, i \neq k}^{K} \phi_{j j k} \frac{1}{N} \overline{\mathbf{h}}_{j j i}^{H} \overline{\mathbf{h}}_{j j i}
\end{aligned}
$$

and $\bar{\theta}_{j}$ si given by (21).

Proof: The proof is omitted for space limitations but relies on the same random matrix tools of those used in Appendix A for Lemma 1.

As for uncorrelated Rayleigh fading channels [7], the asymptotic expressions for MRT and MRC are very similar. The main difference between (17) and (18) is that (18) is only a function of $\bar{\theta}_{j}$ whereas (17) depends on all the factors $\left\{\bar{\theta}_{l} ; l=\right.$ $1, \ldots, L\}$. As it follows from (21), the latter depends on the channel estimation quality through the coefficients $\left\{\phi_{j j k}\right\}$ and also on the normalized inner products $\frac{1}{N} \overline{\mathbf{h}}_{l l k}^{H} \overline{\mathbf{h}}_{l l k}$ of the LoS components in all other cells. Also, the asymptotic expressions (17) and (18) provide some insights into the basic behaviours of Massive MIMO systems with MRT and MRC under Rician fading channels. The signal term in the numerator of (17) and (18) scales quadratically with $\frac{1}{N} \overline{\mathbf{h}}_{j j k}^{H} \overline{\mathbf{h}}_{j j k}$. Following [6], the second term in the denominator is referred to as non-coherent interference because, as for Rayleigh channels, it vanishes with $1 / N$ as $N \rightarrow \infty$ and $K$ is kept fixed [6]. Accordingly, the third term is called coherent interference since it maintains constant with respect to $N$ as the signal term. Unlike for Rayleigh channels, it is not only a consequence of pilot contamination but also of the intracell interference generated by the LoS components. Observe that the coherent interference vanishes when pilot contamination is not present. This occurs when every UE uses a unique orthogonal pilot for channel estimation (with ensuing reduction of the system SE) or advanced pilot decontamination schemes are used [6, Sec. 3.5].

We are ultimately interested in the ergodic achievable $\mathrm{UL}$ rates $r_{j k}^{\mathrm{dl}-\mathrm{MRT}}=\log _{2}\left(1+\gamma_{j k}^{\mathrm{dl}-\mathrm{MRT}}\right)$ and $r_{j k}^{\mathrm{ul}-\mathrm{MRC}}=$ $\mathbb{E}_{\widehat{\mathbf{H}}_{j j}}\left\{\log _{2}\left(1+\gamma_{j k}^{\mathrm{ul}-\mathrm{MRC}}\right)\right\}$. Since the logarithm is a continuous function, by applying the continuous mapping theorem [12], from the almost sure convergence results of Lemma 1 it follows that

$$
r_{j k}^{\mathrm{ul}-\mathrm{MRC}} \asymp \bar{r}_{j k}^{\mathrm{ul}-\mathrm{MRC}}=\log _{2}\left(1+\bar{\gamma}_{j k}^{\mathrm{MRC}}\right) .
$$

Similarly, by applying the continuous mapping theorem and the dominated convergence theorem [12], we have that

$$
r_{j k}^{\mathrm{dl}-\mathrm{MRC}} \asymp \bar{r}_{j k}^{\mathrm{dl}-\mathrm{MRC}}=\log _{2}\left(1+\bar{\gamma}_{j k}^{\mathrm{MRC}}\right) .
$$

\section{B. S-MMSE and RZF}

To begin with, call $\boldsymbol{\Phi}_{j j}=\operatorname{diag}\left\{\phi_{j j 1}, \ldots, \phi_{j j K}\right\}$ and rewrite $\widehat{\mathbf{H}}_{j j}=\left[\widehat{\mathbf{h}}_{j j 1} \cdots \widehat{\mathbf{h}}_{j j K}\right] \in \mathbb{C}^{N \times K}$ as

$$
\widehat{\mathbf{H}}_{j j}=\mathbf{Z}_{j j} \boldsymbol{\Phi}_{j j}+\overline{\mathbf{H}}_{j j} \text {. }
$$

Then, let us introduce the fundamental equations that are needed to express an asymptotic approximation of $\gamma_{j k}$ under S-MMSE and RZF. We start with:

$$
\begin{aligned}
\delta_{j} & =\frac{1}{N} \operatorname{tr}\left(\lambda_{j}\left(1+\widetilde{\delta}_{j}\right) \mathbf{I}_{N}+\frac{1}{N} \overline{\mathbf{H}}_{j j}\left(\mathbf{I}_{K}+\delta_{j} \boldsymbol{\Phi}_{j j}\right)^{-1} \overline{\mathbf{H}}_{j j}^{H}\right)^{-1} \\
& \triangleq \frac{1}{N} \operatorname{tr}\left(\mathbf{T}_{j}\right) \\
\widetilde{\delta}_{j} & =\frac{1}{N} \operatorname{tr}\left(\boldsymbol{\Phi}_{j j}\left(\lambda_{j}\left(\mathbf{I}_{K}+\delta_{j} \boldsymbol{\Phi}_{j j}\right)+\frac{1}{N} \frac{\overline{\mathbf{H}}_{j j}^{H} \overline{\mathbf{H}}_{j j}}{1+\widetilde{\delta}_{j}}\right)^{-1}\right) \\
& \triangleq \frac{1}{N} \operatorname{tr}\left(\boldsymbol{\Phi}_{j j} \widetilde{\mathbf{T}}_{j}\right)
\end{aligned}
$$

which admits a unique positive solution in the class of Stieltjes transforms of non-negative measures with support $\mathbb{R}_{+}$[8], [9]. Notice that the matrices $\mathbf{T}_{j}$ and $\widetilde{\mathbf{T}}_{j}$

$$
\begin{aligned}
& \mathbf{T}_{j}=\left(\lambda_{j}\left(1+\widetilde{\delta}_{j}\right) \mathbf{I}_{N}+\frac{1}{N} \overline{\mathbf{H}}_{j j}\left(\mathbf{I}_{K}+\delta_{j} \boldsymbol{\Phi}_{j j}\right)^{-1} \overline{\mathbf{H}}_{j j}^{H}\right)^{-1} \\
& \widetilde{\mathbf{T}}_{j}=\left(\lambda_{j}\left(\mathbf{I}_{K}+\delta_{j} \boldsymbol{\Phi}_{j j}\right)+\frac{1}{N} \frac{\overline{\mathbf{H}}_{j j}^{H} \overline{\mathbf{H}}_{j j}}{1+\widetilde{\delta}_{j}}\right)^{-1}
\end{aligned}
$$

in (28) and 29) are approximations of the resolvent $\mathbf{Q}_{j j}=$ $\left(\frac{1}{N} \widehat{\mathbf{H}}_{j j} \widehat{\mathbf{H}}_{j j}^{H}+\lambda_{j} \mathbf{I}_{N}\right)^{-1}$ and co-resolvent $\widetilde{\mathbf{Q}}_{j j}=\left(\frac{1}{N} \widehat{\mathbf{H}}_{j j}^{H} \widehat{\mathbf{H}}_{j j}+\right.$ $\left.\lambda_{j} \mathbf{I}_{K}\right)^{-1}$. Also, let us define the following quantities (that will be useful in the remainder of this work):

$$
F_{j}=\left(1+\tilde{\delta}_{j}\right)^{-2} \frac{1}{N^{2}} \operatorname{tr}\left(\boldsymbol{\Phi}_{j j} \widetilde{\mathbf{T}}_{j} \overline{\mathbf{H}}_{j j}^{H} \overline{\mathbf{H}}_{j j} \widetilde{\mathbf{T}}_{j}\right)
$$




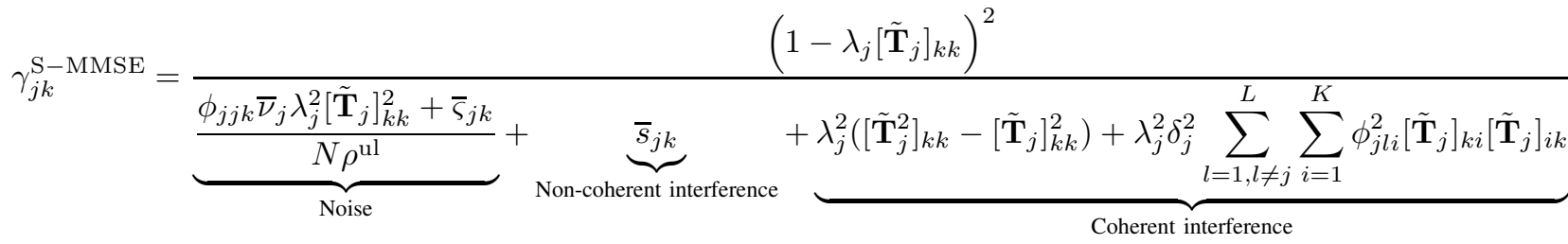

$$
\begin{aligned}
& \gamma_{j k}^{\mathrm{RZF}}=\frac{\bar{\psi}_{j}\left(1-\lambda_{j}\left[\widetilde{\mathbf{T}}_{j}\right]_{k k}\right)^{2}}{\underbrace{\frac{1}{N \rho^{\mathrm{dl}}}}_{\text {Noise }}+\underbrace{\bar{s}_{j k}}_{\text {Non-coherent interference }}+\underbrace{\bar{\psi}_{j} \lambda_{j}^{2}\left(\left[\widetilde{\mathbf{T}}_{j}^{2}\right]_{k k}-\left[\widetilde{\mathbf{T}}_{j}\right]_{k k}^{2}\right)+\sum_{l=1, l \neq j}^{L} \bar{\psi}_{l}\left(\phi_{l j k} \delta_{l}\right)^{2} \lambda_{l}^{2}\left[\widetilde{\mathbf{T}}_{l}^{2}\right]_{k k}}}
\end{aligned}
$$

$$
\begin{gathered}
\Delta_{j}=\left(1-F_{j}\right)^{2}-\lambda_{j}^{2} \frac{1}{N} \operatorname{tr}\left(\mathbf{T}_{j}^{2}\right) \frac{1}{N} \operatorname{tr}\left(\mathbf{\Phi}_{j j} \widetilde{\mathbf{T}}_{j}\right)^{2} \\
\tilde{\vartheta}_{j}=\frac{1}{N} \operatorname{tr}\left(\mathbf{\Phi}_{j j} \widetilde{\mathbf{T}}_{j} \mathbf{\Phi}_{j j} \widetilde{\mathbf{T}}_{j}\right) \\
\bar{\nu}_{j}=\frac{1}{\Delta_{j}} \frac{1}{N} \operatorname{tr}\left(\mathbf{T}_{j}^{2}\right) \\
\bar{\zeta}_{j k}=\frac{1-F_{j}}{\Delta_{j}} \frac{\left[\tilde{\mathbf{T}}_{j} \frac{1}{N} \overline{\mathbf{H}}_{j j}^{H} \overline{\mathbf{H}}_{j j} \tilde{\mathbf{T}}_{j}\right]_{k k}}{\left(1+\tilde{\delta}_{j}\right)^{2}} \\
+\bar{\nu}_{j} \lambda_{j}^{2}\left(\left[\tilde{\mathbf{T}}_{j} \boldsymbol{\Phi}_{j} \tilde{\mathbf{T}}_{j}\right]_{k k}-\phi_{j j k}\left[\tilde{\mathbf{T}}_{j}\right]_{k k}^{2}\right) \\
\xi_{j k}=\bar{\nu}_{j} \lambda_{j}^{2}\left[\tilde{\mathbf{T}}_{j} \boldsymbol{\Phi}_{j} \tilde{\mathbf{T}}_{j}\right]_{k k}+\frac{1-F_{j}}{\Delta_{j}} \frac{\left[\tilde{\mathbf{T}}_{j} \frac{1}{N} \overline{\mathbf{H}}_{j j}^{H} \overline{\mathbf{H}}_{j j} \tilde{\mathbf{T}}_{j}\right]_{k k}}{\left(1+\tilde{\delta}_{j}\right)^{2}} \\
\zeta_{j k}=\frac{1-F_{j}}{\Delta_{j}} \lambda_{j}^{2}\left[\tilde{\mathbf{T}}_{j} \mathbf{\Phi}_{j} \tilde{\mathbf{T}}_{j}\right]_{k k}+\frac{\lambda_{j}^{2} \tilde{\vartheta}_{j}}{\Delta_{j}} \frac{\left[\tilde{\mathbf{T}}_{j} \frac{1}{N} \overline{\mathbf{H}}_{j j}^{H} \overline{\mathbf{H}}_{j j} \tilde{\mathbf{T}}_{j}\right]_{k k}}{\left(1+\tilde{\delta}_{j}\right)^{2}}
\end{gathered}
$$

The following theorems represent a major result of this work.

Theorem 1 (S-MMSE). Let Assumptions 1 - 2 hold true. If S-MMSE is employed, then $\gamma_{j k}^{\mathrm{S}-\mathrm{MMSE}} \asymp \bar{\gamma}_{j k}^{\mathrm{S}-\mathrm{MMSE}}$ with $\bar{\gamma}_{j k}^{\mathrm{S}-\mathrm{MMSE}}$ given by (25) where

$$
\bar{s}_{j k}=\left(\sum_{l=1}^{L} \frac{1}{N} \sum_{i=1}^{K} \mu_{j l i}\right) \xi_{j k}+\sum_{l=1}^{L} \frac{1}{N} \sum_{i=1}^{K} \gamma_{j l i} \zeta_{j k}
$$

with

$\mu_{j l i}=\left\{\begin{array}{cc}d_{j j i}-\phi_{j j i}+\lambda_{j}^{2}\left[\tilde{\mathbf{T}}_{j} \boldsymbol{\Phi}_{j} \tilde{\mathbf{T}}_{j}\right]_{i i} & l=j \\ d_{j l i}-\lambda_{j} \phi_{j l i}^{2} \delta_{j}\left(2\left[\tilde{\mathbf{T}}_{j}\right]_{i i}+\delta_{j} \lambda_{j}\left[\tilde{\mathbf{T}}_{j} \boldsymbol{\Phi}_{j} \tilde{\mathbf{T}}_{j}\right]_{i i}\right) & l \neq j\end{array}\right.$

and

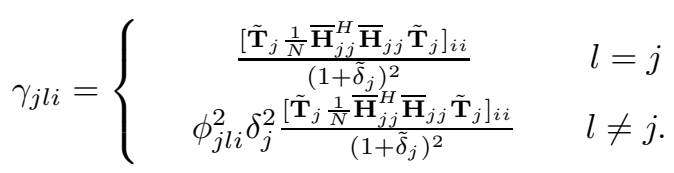

Proof: The proof is sketched in Appendix B and relies heavily on the techniques developed in [8] and [37]. The presence of channel estimation errors and pilot contamination makes it necessary to apply those techniques to new random quantities whose computation in explicit form requires lengthy derivations. A large effort has been made to present the results in a simple form.
Theorem 2 (RZF). Let Assumptions $1-2$ hold true. If RZF is employed, then $\gamma_{j k}^{\mathrm{RZF}} \asymp \bar{\gamma}_{j k}^{\mathrm{RZF}}$ with $\bar{\gamma}_{j k}^{\mathrm{RZF}}$ given by (26) where $\bar{\psi}_{j}$ takes the form:

$\bar{\psi}_{j}=\left(\lambda_{j}^{2} \bar{\nu}_{j} \frac{1}{K} \operatorname{tr} \boldsymbol{\Phi}_{j j} \widetilde{\mathbf{T}}_{j}^{2}+\frac{1-F_{j}}{\Delta_{j}\left(1+\tilde{\delta}_{j}\right)^{2}} \frac{1}{K N} \operatorname{tr} \widetilde{\mathbf{T}}_{j} \overline{\mathbf{H}}_{j j}^{H} \overline{\mathbf{H}}_{j j} \widetilde{\mathbf{T}}_{j}\right)^{-1}$

and

$$
\bar{s}_{j k}=\sum_{l=1}^{L} \bar{\psi}_{l} \mu_{l j k} \frac{1}{N} \sum_{i=1}^{K} \xi_{i l}+\sum_{l=1}^{L} \bar{\psi}_{l} \gamma_{l j k} \frac{1}{N} \sum_{i=1}^{K} \zeta_{l i} .
$$

Proof: The proof is omitted for space limitations, but follows along the lines of Theorem 1 .

Unlike the asymptotic expressions for MRC and MRT, those provided in Theorems 1 and 2 are much more involved. The distinction between non-coherent interference and coherent terms is still doable and provides evidence of the fact that the latter depends through complicated expressions of the intracell LoS components $\left\{\overline{\mathbf{h}}_{j j i} ; i=1, \ldots, K\right\}$. Despite being involved, when applied to practical networks such approximations are very much useful since they can be used to simulate the network behavior under different settings without to carry out extensive Monte-Carlo simulations. In fact, numerical results provided in Section $\mathrm{V}$ prove that the approximations provided in Theorems 2 and 11 are asymptotically tight, but also accurate for systems with finite dimensions. Moreover, as exemplified in the sequel and in Section IV they can be used to get important insights, with respect to CSI quality, induced interference and impact of LoS components.

\section{Limiting case $N \rightarrow \infty$ with $K / N \rightarrow 0$}

We now look at the limiting case in which $N \rightarrow \infty$ such that $K / N \rightarrow 0$. The following results are easily obtained from the asymptotic analysis above:

Corollary 1 (MRC and MRT). If $N \rightarrow \infty$ such that $K / N \rightarrow$ 0 , then $\bar{\gamma}_{j k}^{\mathrm{MRC}}$ reduces to:

$$
\bar{\gamma}_{j k}^{\mathrm{MRC}}=\underbrace{\frac{\left(\phi_{j j k}+\frac{1}{N} \overline{\mathbf{h}}_{j j k}^{H} \overline{\mathbf{h}}_{j j k}\right)^{2}}{\sum_{i=1, i \neq k}^{K}\left|\frac{1}{N} \overline{\mathbf{h}}_{j j i}^{H} \overline{\mathbf{h}}_{j j k}\right|^{2}+\sum_{l=1, l \neq j}^{L} \phi_{l j k}^{2}}}_{\text {Coherent interference }} .
$$


Also, $\bar{\gamma}_{j k}^{\mathrm{MRT}}$ becomes

$$
\bar{\gamma}_{j k}^{\mathrm{MRT}}=\underbrace{\frac{\left(\phi_{j j k}+\frac{1}{N} \overline{\mathbf{h}}_{j j k}^{H} \overline{\mathbf{h}}_{j j k}\right)^{2}}{\sum_{i=1, i \neq k}^{K}\left|\frac{1}{N} \overline{\mathbf{h}}_{j j i}^{H} \overline{\mathbf{h}}_{j j k}\right|^{2}+\sum_{l=1, l \neq j}^{L} \frac{\bar{\theta}_{l}}{\bar{\theta}_{j}} \phi_{l j k}^{2}}}_{\text {Coherent interference }}
$$

with $\bar{\theta}_{j}$ given by (21).

Proof: The proof follows easily from Lemmas 1 and 2 by noticing that the non-coherent interference $\bar{s}_{j k} \asymp$ $\bar{\theta}_{j} \sum_{i=1, i \neq k}^{K}\left|\frac{1}{N} \overline{\mathbf{h}}_{j j i}^{H} \overline{\mathbf{h}}_{j j k}\right|^{2}$ as $N \rightarrow \infty$ with $K / N \rightarrow 0$.

The above corollaries show that when $N$ grows at a faster rate than $K$, differently from Rayleigh fading, the coherent interference depends also on the asymptotic behavior of the inner products $\frac{1}{N} \overline{\mathbf{h}}_{j j i}^{H} \overline{\mathbf{h}}_{j j k}$. If the BS is equipped with a uniform linear array (ULA) and LoS vectors $\overline{\mathbf{h}}_{j j i}$ and $\overline{\mathbf{h}}_{j j k}$ are either aligned in the complex plane or have an angular difference that scales as $1 / M^{\alpha}$ with $\alpha \geq 1$, then $\frac{1}{N} \overline{\mathbf{h}}_{j j i}^{H} \overline{\mathbf{h}}_{j j k}$ does not vanish asymptotically. Both cases belong to the category of scenarios for which the favorable propagation conditions are not satisfied [23], [38]. Similar observations can be made under RZF and S-MMSE, as shown next.

Corollary 2 (S-MMSE and RZF). If $N \rightarrow \infty$ such that $K / N \rightarrow 0$, we have that $\bar{\gamma}_{j k}^{\mathrm{S}-\mathrm{MMSE}}$ reduces to

$$
\underbrace{\frac{\left(1-\lambda_{j}\left[\tilde{\mathbf{T}}_{j}\right]_{k k}\right)^{2}}{\lambda_{j}^{2}\left(\left[\tilde{\mathbf{T}}_{j}^{2}\right]_{k k}-\left[\tilde{\mathbf{T}}_{j}\right]_{k k}^{2}\right)+\sum_{l=1, l \neq j}^{L} \sum_{i=1}^{K} \phi_{j l i}^{2}\left[\tilde{\mathbf{T}}_{j}\right]_{k i}\left[\tilde{\mathbf{T}}_{j}\right]_{i k}}}_{\text {Coherent interference }}
$$

with

$$
\widetilde{\mathbf{T}}_{j}=\left(\lambda_{j} \mathbf{I}_{K}+\boldsymbol{\Phi}_{j j}+\frac{1}{N} \overline{\mathbf{H}}_{j j}^{H} \overline{\mathbf{H}}_{j j}\right)^{-1} .
$$

Also, $\bar{\gamma}_{j k}^{\mathrm{RZF}}$ reduces to

$$
\underbrace{\frac{\bar{\psi}_{j}\left(1-\lambda_{j}\left[\widetilde{\mathbf{T}}_{j}\right]_{k k}\right)^{2}}{\bar{\psi}_{j} \lambda_{j}^{2}\left(\left[\widetilde{\mathbf{T}}_{j}^{2}\right]_{k k}-\left[\widetilde{\mathbf{T}}_{j}\right]_{k k}^{2}\right)+\sum_{l=1, l \neq j}^{L} \bar{\psi}_{l} \phi_{l j k}^{2}\left[\widetilde{\mathbf{T}}_{l}^{2}\right]_{k k}}}_{\text {Coherent interference }}
$$

where $\bar{\psi}_{j}=\left(\frac{1}{K} \operatorname{tr} \boldsymbol{\Phi}_{j j} \widetilde{\mathbf{T}}_{j}^{2}+\frac{1}{K N} \operatorname{tr} \widetilde{\mathbf{T}}_{j} \overline{\mathbf{H}}_{j j}^{H} \overline{\mathbf{H}}_{j j} \widetilde{\mathbf{T}}_{j}\right)^{-1}$.

Proof: The proof relies on observing that if $N \rightarrow \infty$ with $K / N \rightarrow 0$ then $\delta_{j} \rightarrow \lambda_{j}^{-1}$ and $\frac{1}{N} \operatorname{tr} \mathbf{T}_{j}^{2} \rightarrow \lambda_{j}^{-2}$. Also, $F_{j} \rightarrow 0, \Delta_{j} \rightarrow 1$ and $\bar{\nu}_{j} \rightarrow \lambda_{j}^{-2}$. Using these results into the expressions in Theorems 1 and 2 completes the proof.

\section{Limiting case $N \rightarrow \infty$ with $K / N \rightarrow 0$ under favorable propagations}

Consider now a system in which the favorable propagation conditions are asymptotically satisfied, i.e., $\frac{1}{N} \overline{\mathbf{h}}_{j j i}^{H} \overline{\mathbf{h}}_{j j k} \rightarrow 0$ $\forall i \neq k$ as $N \rightarrow \infty$ [23], [38]. For simplicity, we only consider MRT and RZF, but similar results are obtained for MRC and S-MMSE. Then, we have that:

Corollary 3. If $N \rightarrow \infty$ with $K / N \rightarrow 0$ and $\frac{1}{N} \overline{\mathbf{h}}_{j j i}^{H} \overline{\mathbf{h}}_{j j k} \rightarrow 0$ $\forall i \neq k$, then:

$$
\bar{\gamma}_{j k}^{\mathrm{MRT}}=\frac{\bar{\theta}_{j}\left(\phi_{j j k}+\frac{1}{N} \overline{\mathbf{h}}_{j j k}^{H} \overline{\mathbf{h}}_{j j k}\right)^{2}}{\sum_{l=1, l \neq j}^{L} \bar{\theta}_{l} \phi_{l j k}^{2}}
$$

with $\bar{\theta}_{j}$ given by 21 .

Corollary 4 (RZF). If $N \rightarrow \infty$ with $K / N \rightarrow 0$ and $\frac{1}{N} \overline{\mathbf{h}}_{j j i}^{H} \overline{\mathbf{h}}_{j j k} \rightarrow 0 \forall i \neq k$, then:

$$
\begin{aligned}
\bar{\gamma}_{j k}^{\mathrm{RZF}} & =\frac{\bar{\psi}_{j}\left(\frac{\phi_{j j k}+\frac{1}{N} \overline{\mathbf{h}}_{j j k}^{H} \overline{\mathbf{h}}_{j j k}}{\lambda_{j}+\phi_{j j k}+\frac{1}{N} \overline{\mathbf{h}}_{j j k}^{H} \mathbf{\overline { h }}_{j j k}}\right)^{2}}{\sum_{l=1, l \neq j}^{L} \bar{\psi}_{l}\left(\frac{\phi_{l j k}}{\lambda_{l}+\phi_{l l k}+\frac{1}{N} \overline{\mathbf{h}}_{l l k}^{H} \overline{\mathbf{h}}_{l l k}}\right)^{2}} \\
& =\frac{\bar{\psi}_{j}\left(\phi_{j j k}+\frac{1}{N} \overline{\mathbf{h}}_{j j k}^{H} \overline{\mathbf{h}}_{j j k}\right)^{2}}{\sum_{l=1, l \neq j}^{L}\left(\frac{\lambda_{j}+\phi_{j j k}+\frac{1}{N} \overline{\mathbf{h}}_{j k}^{H} \overline{\mathbf{h}}_{j j k}}{\lambda_{l}+\phi_{l l k}+\frac{1}{N} \overline{\mathbf{h}}_{l l k}^{H} \overline{\mathbf{h}}_{l l k}}\right)^{2} \bar{\psi}_{l} \phi_{l j k}^{2}}
\end{aligned}
$$

with $\psi_{j}$ given by

$$
\bar{\psi}_{j}=\left(\frac{1}{K} \sum_{k=1}^{K} \frac{\phi_{j j k}+\frac{1}{N} \overline{\mathbf{h}}_{j j k}^{H} \overline{\mathbf{h}}_{j j k}}{\left(\lambda_{j}+\phi_{j j k}+\frac{1}{N} \overline{\mathbf{h}}_{j j k}^{H} \overline{\mathbf{h}}_{j j k}\right)^{2}}\right)^{-1} .
$$

Proof: If $\frac{1}{N} \overline{\mathbf{h}}_{j j i}^{H} \overline{\mathbf{h}}_{j j k} \rightarrow 0 \forall i \neq k$, then $\widetilde{\mathbf{T}}_{j}$ in (47) is diagonal with $\left[\widetilde{\mathbf{T}}_{j}\right]_{k k}=\left(\lambda_{j}+\phi_{j j k}+\frac{1}{N} \overline{\mathbf{h}}_{j j k}^{H} \overline{\mathbf{h}}_{j j k}\right)^{-1}$.

In line with [19], [38], the above corollaries show that if the uncorrelated Rician fading channels result in favorable propagations, then the interference vanishes as $N$ grows unbounded for both MRT and RZF. In practice, this means that those asymptotic values can only be achieved if some UEs are dropped from service [10], [38]. From Corollaries 3 and 4, it also turns out that, as for Rayleigh fading channels [7. Corollary 1], the asymptotic SINRs under RZF and MRT are not necessarily the same. This is because the matrix $\mathbf{Q}_{j}$ with RZF depends on the correlation matrix $\boldsymbol{\Phi}_{j j}$ through (27).

\section{ON THE EFFECT OF LOS COMPONENTS: A CASE STUDY}

To get further insights on the effect of LoS components, we consider the regime in which $N$ and $K$ grow to infinity at the same pace and assume that the channel can be simply modelled as:

$$
\mathbf{h}_{j l k}=\left\{\begin{array}{cc}
\sqrt{\frac{1}{1+\kappa}} \mathbf{z}_{j j k}+\sqrt{\frac{\kappa}{1+\kappa}} \mathbf{a}_{j j k} & l=j \\
\sqrt{\alpha} \mathbf{z}_{j l k} & l \neq j
\end{array}\right.
$$

where $\alpha \in(0,1]$ is the intercell interference factor and $\kappa$ is the Rician factor, which is assumed to be the same for all UEs in cell $j$. Using the above model, we have that

$$
\phi_{j l k}=\left\{\begin{array}{cc}
\frac{1}{(1+\kappa)^{2}} \nu & l=j \\
\frac{\alpha}{1+\kappa} \nu & l \neq j
\end{array}\right.
$$




$$
\vartheta=-\frac{N}{K} \frac{\kappa}{(1+\kappa)} \frac{-\left(\tilde{\delta}^{\star}\right)^{2} \lambda-\tilde{\delta}^{\star}\left(\lambda+\phi\left(1-\frac{3 K}{N}\right)+\frac{\kappa}{1+\kappa}+\frac{K}{N} \phi^{2} \frac{1+\kappa}{\kappa}\right)+\phi \frac{K}{N}-\phi^{2} \frac{1+\kappa}{\kappa} \frac{K}{N}}{\lambda^{2} \phi^{3}\left(1+\tilde{\delta}^{\star}\right)^{3}}
$$

with $\nu=\frac{\rho^{\operatorname{tr}}}{1+\rho^{\operatorname{tr}} \bar{L}}$ and $\bar{L}=\alpha(L-1)+\frac{1}{1+\kappa}$. We assume that $\overline{\mathbf{H}}_{j j}$ has orthogonal columns such that the LoS matrix $\overline{\mathbf{H}}_{j j}$ is unitary, $\frac{1}{N} \overline{\mathbf{H}}_{j j}^{H} \overline{\mathbf{H}}_{j j}=\frac{\kappa}{1+\kappa} \mathbf{I}_{K}$. This is achieved if the vectors $\mathbf{a}_{j j k}$ are such that $\frac{1}{N} \mathbf{a}_{j j k}^{H} \mathbf{a}_{j j k}=1$ and $\frac{1}{N} \mathbf{a}_{j j i}^{H} \mathbf{a}_{j j k}=0$ $\forall i \neq k$. As mentioned before, in practical networks this means that some UEs must be dropped from service [10], [38].

Corollary 5 (MRC and MRT). Let Assumptions $1-2$ hold true. If the channel is modeled as in (53) and $\frac{1}{N} \overline{\mathbf{H}}_{j j}^{H} \overline{\mathbf{H}}_{j j}=$ $\frac{\kappa}{1+\kappa} \mathbf{I}_{K} \forall j$, then $\bar{\gamma}_{j k}^{\mathrm{MRC}}$ and $\bar{\gamma}_{j k}^{\mathrm{MRT}}$ reduce both to

$$
\begin{aligned}
& \frac{1}{\nu N \rho^{\mathrm{dI}} \frac{1+\kappa}{\tau}+\frac{K}{N \nu}\left(\bar{L} \frac{1+\kappa}{\tau}+\frac{1}{\tau^{2}} \frac{\kappa}{1+\kappa}\right)+\frac{\alpha}{\tau^{2}}\left(\bar{L}-\frac{1}{1+\kappa}\right)} \\
& =\frac{1}{\underbrace{\frac{L}{N \rho^{\mathrm{dl}}} \frac{1+\kappa}{\tau}}_{\text {Noise }}+\underbrace{\frac{1}{\rho^{\mathrm{tr}}} A}_{\text {Imperfect CSI }}+\underbrace{\frac{K}{N} \bar{L} B}_{\text {Interference }}+\underbrace{\frac{\alpha}{\tau^{2}}\left(\bar{L}-\frac{1}{1+\kappa}\right)}_{\text {Pilot Contamination }}}
\end{aligned}
$$

where $\nu=\frac{\rho^{\operatorname{tr}}}{1+\rho^{\operatorname{tr}} \bar{L}}$ and $\bar{L}=\alpha(L-1)+\frac{1}{1+\kappa}$ and

$$
\begin{aligned}
\tau & =\frac{1}{1+\kappa}+\frac{\kappa}{\nu} \\
A & =\left(\frac{K}{N} \bar{L}+\frac{1}{N \rho^{\mathrm{dl}}}\right) \frac{1+\kappa}{\tau}+\frac{K}{N} \frac{1}{\tau^{2}} \frac{\kappa}{1+\kappa} \\
B & =\bar{L} \frac{1+\kappa}{\tau}+\frac{1}{\tau^{2}} \frac{\kappa}{1+\kappa} .
\end{aligned}
$$

Proof: Consider the asymptotic results for MRT in (17). If the channel is modeled as in (53) and $\frac{1}{N} \overline{\mathbf{H}}_{j j}^{H} \overline{\mathbf{H}}_{j j}=\frac{\kappa}{1+\kappa} \mathbf{I}_{K}$, then $\bar{\theta}_{j}\left(\phi_{j j k}+\frac{1}{N} \overline{\mathbf{h}}_{j j k}^{H} \overline{\mathbf{h}}_{j j k}\right)^{2}$ reduces to $\tau \frac{\nu}{1+\kappa}$ whereas the coherent interference becomes $\frac{\alpha^{2}}{\tau} \frac{\nu}{1+\kappa}(L-1)$. On the other hand, the non-coherent interference reduces to $\frac{K}{N}(\alpha(L-1)+$ $\left.\frac{1}{1+\kappa}+\frac{1}{\tau} \frac{\kappa}{(1+\kappa)^{2}}\right)$. Putting these results together yields (55) from which (56) follows. Notice that if $\kappa=0$ then $\bar{\gamma}_{j k}^{\mathrm{MRC}}$ and $\bar{\gamma}_{j k}^{\mathrm{MRT}}$ coincide with the expressions provided in [7, Cor. 2].

Corollary 6 (S-MMSE and RZF). Let Assumptions 1 - 2 hold true. Denote $\widetilde{\delta}^{\star}$ the real positive solution of the following thirdorder polynomial equation

$$
\begin{aligned}
\lambda x^{3} & +x^{2}\left(2 \lambda+\phi\left(1-\frac{K}{N}\right)\right) \\
& +x\left(\lambda+\phi\left(1-2 \frac{K}{N}\right)+\frac{\kappa}{1+\kappa}\right)-\phi \frac{K}{N}=0
\end{aligned}
$$

with $\lambda=\frac{K}{N}\left(\alpha(L-1)+\frac{1}{1+\kappa}-\phi\right)$ and $\phi=\frac{\nu}{(1+\kappa)^{2}}$. Define $\vartheta$ as in (49) on the top of the page and

$$
\Delta=\left(1-\frac{\kappa}{1+\kappa} \frac{N}{K \phi} \frac{\left(\tilde{\delta}^{\star}\right)^{2}}{\left(1+\tilde{\delta}^{\star}\right)^{2}}\right)^{2}-\lambda^{2} \vartheta \frac{N}{K}\left(\tilde{\delta}^{\star}\right)^{2} .
$$

Let $\delta^{\star}=\frac{\tilde{\delta}^{\star}}{\phi}+\frac{1-K / N}{\lambda}$. If the channel is modeled as in (53) and $\frac{1}{N} \overline{\mathbf{H}}_{j j}^{H} \overline{\mathbf{H}}_{j j}=\frac{\kappa}{1+\kappa} \mathbf{I}_{K} \forall j$, then $\gamma_{j k}^{\mathrm{RZF}}$ and $\bar{\gamma}_{j k}^{\mathrm{S}-\mathrm{MMSE}}$ reduce both to

$$
\frac{1}{\frac{1}{\nu N \rho^{\mathrm{d}}} \frac{1+\kappa}{X}+\bar{s}^{\prime}+\frac{\alpha}{\underline{\tau}^{2}}\left(\bar{L}-\frac{1}{1+\kappa}\right)}
$$

where $X^{-1}=\frac{N}{K}\left(-1+\frac{1-\frac{\kappa(1+\kappa)}{\nu} \frac{N}{K}\left(\tilde{\delta}^{\star}\right)^{2}}{\Delta}\right) \frac{1+\kappa}{\left(1-\lambda \frac{N}{K} \frac{\tilde{\delta}^{\star}}{\phi}\right)^{2}}, \bar{s}^{\prime}=$ $\frac{\bar{s}}{\bar{\psi}\left(1-\lambda \frac{N}{K} \frac{\tilde{\delta}^{\star}}{\phi}\right)^{2}}$ with $\frac{\bar{s}}{\bar{\psi}}$ given in Appendix $C$, and

$$
\underline{\tau}=\frac{1}{1+\kappa}+\frac{\kappa}{\nu} \frac{1}{\lambda \delta^{\star}\left(1+\widetilde{\delta}^{\star}\right)}
$$

Proof: The proof sketch is reported in Appendix C.

The above results can be used to get instrumental insights. Let's consider for simplicity the asymptotic expression provided in Corollary 5. As seen, the effective SNR, given by $\nu N \rho^{\mathrm{dl}} \frac{\tau}{1+\kappa}$, increases linearly with $N$ as it happens for uncorrelated Rayleigh fading channels [7, Corollary 2]. Also, it increases with the Rician component $\kappa$ as $\nu \frac{\tau}{1+\kappa}$; notice that $\tau$ increases as $\kappa$ grows large. As for a Rayleigh model, the channel estimation errors and interference vanish only if $N$ grows large. Indeed, if $\kappa$ increases $A$ tends to $\frac{K}{N} \bar{L}+\frac{1}{N \rho^{\text {dr }}}$ whereas $B$ goes to $\bar{L}$. On the other hand, the pilot contamination term goes to zero with $\kappa$ as $1 / \kappa^{2}$ (since $\tau \rightarrow \kappa / \nu$ as $\kappa$ grows large). As for uncorrelated Rayleigh fading channels, in the limiting case in which $N \rightarrow \infty$ such that $K / N \rightarrow 0$, pilot contamination remains the only performance limitation. Unlike in Rayleigh fading channels, however, it depends on the Rician factor as shown below.

Corollary 7. If $N \rightarrow \infty$ with $K / N \rightarrow 0$, then $\bar{\gamma}_{j k}^{\mathrm{MRC}}=$ $\bar{\gamma}_{j k}^{\mathrm{MRT}}=\bar{\gamma}_{j k}^{\mathrm{S}-\mathrm{MMSE}}=\bar{\gamma}_{j k}^{\mathrm{RZF}} \asymp \gamma_{\infty}$ with

$$
\gamma_{\infty}=\frac{1}{\frac{\alpha}{\tau^{2}}\left(\bar{L}-\frac{1}{1+\kappa}\right)}=\frac{\left(\frac{1}{1+\kappa}+\frac{\kappa}{\nu}\right)^{2}}{\alpha^{2}(L-1)}
$$

and the ultimately achievable rate is given by

$$
R_{\infty}=\log _{2}\left(1+\gamma_{\infty}\right)=\log _{2}\left(1+\frac{\left(\frac{1}{1+\kappa}+\frac{\kappa}{\nu}\right)^{2}}{\alpha^{2}(L-1)}\right) .
$$

Proof: It follows from Corollaries 5 and 6 by taking the limit $N \rightarrow \infty$ with $K / N \rightarrow 0$. Specifically, with S-MMSE and RZF, if $K / N \rightarrow 0$ then $\widetilde{\delta}^{\star} \rightarrow 0$ and $\delta^{\star}=\frac{\tilde{\delta}^{\star}}{\phi}+\frac{1-K / N}{\lambda} \rightarrow$ $\lambda$. Also, we have that $X \rightarrow 1$ and $\bar{s}^{\prime} \rightarrow 0$. Moreover, $\underline{\tau}$ in (63) tends to $\frac{1}{1+\kappa}+\frac{\kappa}{\nu}$. This completes the proof.

Notice that if $\kappa=0$ then $R_{\infty}=\log _{2}\left(1+\frac{1}{\alpha^{2}(L-1)}\right)$ coincides with the ultimately achievable rate provided in 


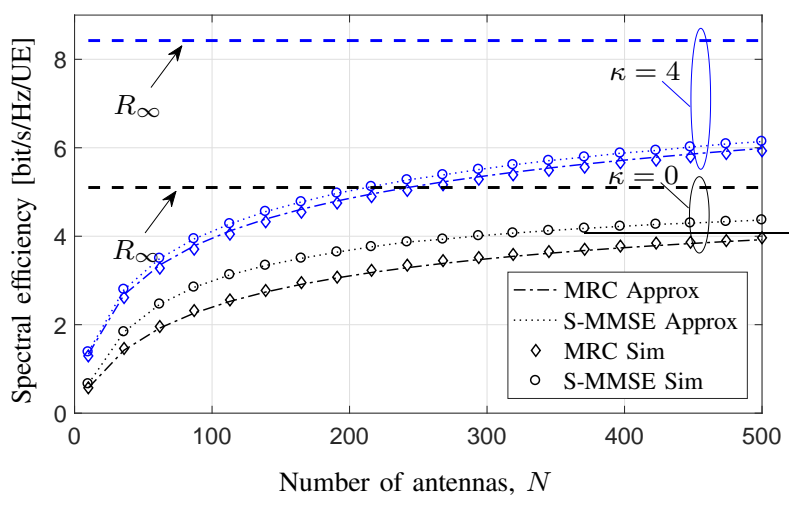

Fig. 1. SE per UE with MRC and S-MMSE as a function of the number of antennas $N$ for the simplified channel given in 53 with $L=4, K=10$, $\alpha=0.1, \rho^{\operatorname{tr}}=6 \mathrm{~dB}, \rho=10 \mathrm{~dB}$, and $\kappa=0$ or 4 .

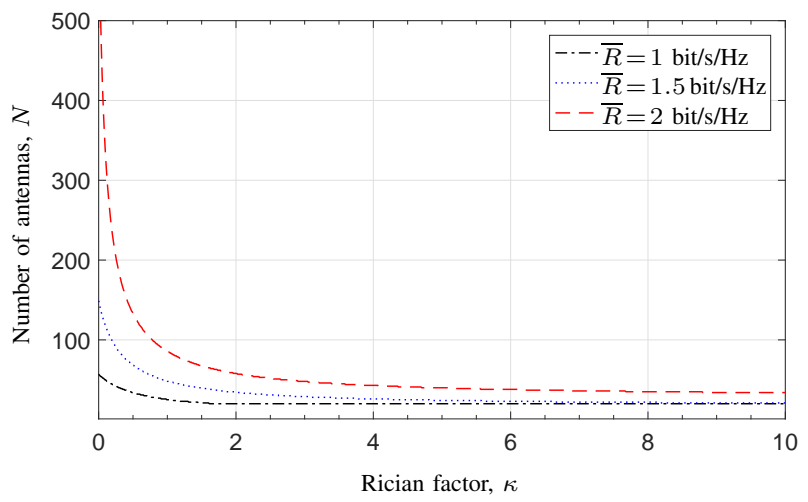

Fig. 2. Number of antennas needed with MRC and the simplified channel given in 53 to achieve SE per UE of $\bar{R} \mathrm{bit} / \mathrm{s} / \mathrm{Hz} / \mathrm{UE}$ versus the Rician factor $\kappa$ with $L=4, K=10, \rho^{\mathrm{tr}}=6 \mathrm{~dB}, \rho=10 \mathrm{~dB}$ and $\alpha=0.3$.

[7. Eq. (32)]. On the other hand, if $\kappa \gg 1$ we have that $\frac{1}{1+\kappa}+\frac{\kappa}{\nu} \approx \frac{1+\rho^{\text {tr }} \alpha(L-1)}{\rho^{\mathrm{tr}}} \kappa$ since $\nu \approx \frac{\rho^{\text {tr }}}{1+\rho^{\mathrm{tr}} \alpha(L-1)}$ and thus

$$
R_{\infty} \approx \log _{2}\left(1+\left(\frac{1}{\rho^{\text {tr }} \alpha}+L-1\right)^{2} \frac{\kappa^{2}}{L-1}\right)
$$

scales logarithmically with $\kappa$ as $2 \log _{2}(\kappa)$.

Before proceeding further, let's us validate the accuracy of the approximations provided in Corollaries 5 and 6 To this end, we assume that the antenna array is uniform and linear with half-wavelength antenna spacing and model $\mathbf{a}_{j j k}$ as $\mathbf{a}_{j j k}=\left[1, e^{-i \pi \sin \left(\vartheta_{j j k}\right)}, \ldots, e^{-i \pi(N-1) \sin \left(\vartheta_{j j k}\right)}\right]^{T}$ where $\vartheta_{j j k}$ denotes the azimuth angle to UE $k$ in any cell $j$. Following [38], we assume that the each BS can create $N$ orthogonal beams with angles $\left\{\vartheta_{j j k}\right\}$ such $\sin \left(\vartheta_{j j k}\right)=-1+\frac{2 k-1}{N}$ and assume that each one of the $K$ UEs is randomly and independently assigned to one of them.

Fig. 1 reports the average rate per UE of MRC and SMMSE as a function of $N$ for $L=4, K=10, \alpha=0.1$, $\rho^{\mathrm{tr}}=6 \mathrm{~dB}, \rho=10 \mathrm{~dB}$, and $\kappa=0$ or 4 . Markers are obtained using Monte-Carlo (MC) simulations whereas the lines are obtained using the closed-form approximations of Corollaries 5 and 6 As seen, the approximations match perfectly with the MC simulations for any $N$. This proves

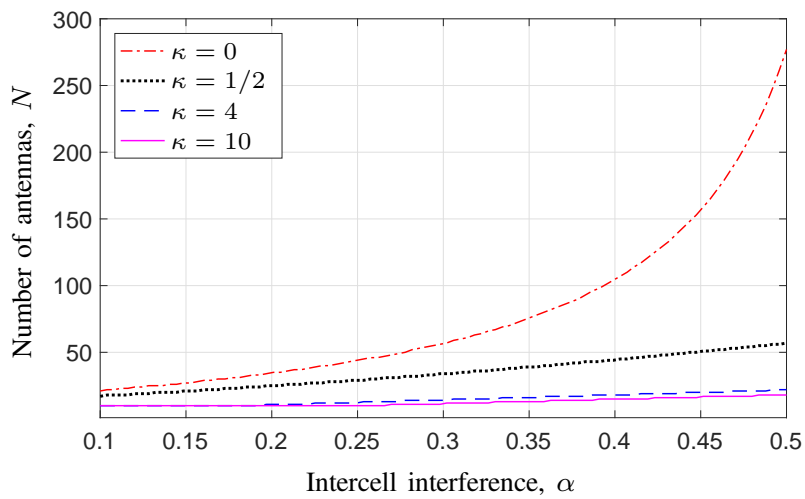

Fig. 3. Number of antennas needed with $\mathrm{MRC}$ and the simplified channel given in $(53)$ to achieve a SE per UE of $\bar{R}=1 \mathrm{bit} / \mathrm{s} / \mathrm{Hz} / \mathrm{UE}$ versus the intercell interference factor $\alpha$ with $L=4, K=10, \rho^{\operatorname{tr}}=6 \mathrm{~dB}, \rho=10 \mathrm{~dB}$.

that the asymptotic approximations are not only asymptotically tight, but accurate even for networks of finite size. Both schemes provide higher rates when $\kappa=4$. As expected, SMMSE outperforms MRC. This is achieved at the price of a higher computational complexity. With $\kappa=4$, the gain of SMMSE is only $3-6 \%$. This means that LoS components may allow not only to achieve higher rates but also to use schemes with lower complexity. As predicted by the analytical results, $R_{\infty}$ increases as $\kappa$ grows. With $\kappa=4, R_{\infty}$ is increased by a factor 1.65 compared to the Rayleigh fading case (i.e., $\kappa=0$ ). However, a larger number of antennas is needed to approach $R_{\infty}$ when $\kappa$ increases. With $N=500$, the S-MMSE achieves $85 \%$ and $70 \%$ of $R_{\infty}$ with $\kappa=0$ and 4 , respectively.

Fig. 2 shows the number of antennas $N$ that is needed with MRC to achieve a given spectral efficiency of $\bar{R} \mathrm{bit} / \mathrm{s} / \mathrm{Hz}$ per UE. We consider $L=4, K=10, \rho^{\mathrm{tr}}=6 \mathrm{~dB}, \rho=10 \mathrm{~dB}$ and an intercell interference factor $\alpha$ of 0.1 or 0.3 . The curves are obtained using the closed-form approximation of Corollary 5 and show the impact of the LoS components in reducing $N$. Compared to the Rayleigh fading case (i.e., $\kappa=0$ ), when $\bar{R}=2 \mathrm{bit} / \mathrm{s} / \mathrm{Hz}, \kappa=4$ and $\alpha=0.3, N$ can be roughly reduced by a factor of 10 . Fig. 3 illustrates the impact of the LoS components when the intercell interference increases in the same setting of Fig. 2 for $\bar{R}=1 \mathrm{bit} / \mathrm{s} / \mathrm{Hz}$. Compared to the case with $\kappa=0$ where an exponential increase of $N$ is observed as $\alpha$ grows, a relatively slow increase is observed in the presence of LoS components. A Rician coefficient of $\kappa=1 / 2$ is enough to reduce the number of antennas of a factor ranging from 1.25 to 4.5 .

\section{Numerical VALIDATION OF THE ASYMPTOTIC ANALYSIS}

MC simulations are now used to validate the accuracy of the above asymptotic analysis of Lemma 1 and Theorem 2 for finite values of $N$ and $K$. The Matlab code available online at https://github.com/lucasanguinetti/ enables further testing. Similar trends are obtained for the UL but are omitted for space limitations. We consider a multicell system with $L=4$ cells, with each covering a square area of $250 \times 250 \mathrm{~m}$. A wrap around topology is used to 


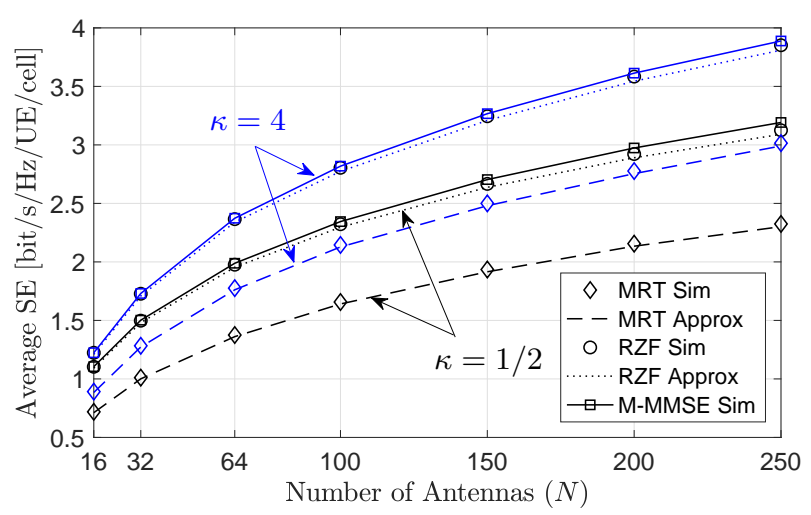

Fig. 4. SE per UE with MRT and RZF versus $N$ when $L=4, K=10$, and the Rician factor is the same for all UEs and equal to $\kappa=1 / 2$ and 4 .

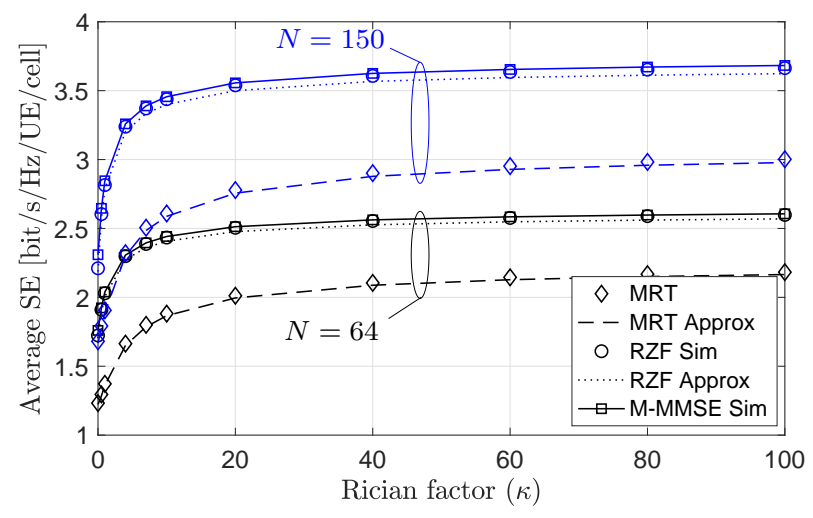

Fig. 5. SE per UE with MRT and RZF vs $\kappa$ when $L=4, K=10$ and $N=64$ or 150 .

simulate that all BSs receive equally much interference from all directions. The parameter $\beta_{j l k}$ is modeled in decibels as $\beta_{j l k}^{j}=\Upsilon-10 \alpha \log _{10}\left(\frac{x_{j l k}}{1 \mathrm{~km}}\right)+\Psi_{j l k}$ where $x_{j l k}[\mathrm{~km}]$ is the distance between the transmitter and the receiver, the pathloss exponent $\alpha=3.7$ determines how fast the signal power decays with the distance, and $\Upsilon=-148 \mathrm{~dB}$ determines the median channel gain at a reference distance of $1 \mathrm{~km}$. Also, $\Psi_{j l k} \sim \mathcal{N}\left(0, \sigma_{\mathrm{sf}}^{2}\right)$ with $\sigma_{\mathrm{sf}}=10$ accounts for the shadow fading. We assume that $K=10$ UEs are randomly and uniformly distributed in each cell, at distances larger than $35 \mathrm{~m}$ from the BS. Results are averaged over 50 UE distributions. We consider communication over a $20 \mathrm{MHz}$ bandwidth with a total receiver noise power of $-94 \mathrm{dBm}$. The median SNR of a UE at $35 \mathrm{~m}$ from its serving BS is $20.6 \mathrm{~dB}$, while a UE in any of the corners of a square cell gets $-5.8 \mathrm{~dB}$. We consider a uniform linear array with half-wavelength antenna spacing for which $\mathbf{a}_{j j k}=\left[1, e^{-i \pi \sin \left(\vartheta_{j j k}\right)}, \ldots, e^{-i \pi(N-1) \sin \left(\vartheta_{j j k}\right)}\right]^{T}$. The angles $\left\{\vartheta_{j j k}\right\}$ are randomly and independently chosen in the interval $[0,2 \pi]$. For simplicity, the Rician factor is the same for all UEs, i.e., $\kappa_{j k}=\kappa \forall j, k$.

Fig. 5illustrates the average SE per UE when $N$ grows large with MRT and RZF. For completeness, comparisons are made with M-MMSE precoding [5], [6]. The Rician factor for all UEs is $\kappa=1 / 2$ and 4 ; that is, the LoS vectors are respectively
$-3 \mathrm{~dB}$ and $6 \mathrm{~dB}$ stronger than Rayleigh vectors. As seen, the asymptotic results perfectly match the MC simulations. RZF provides higher SE than MRT and achieves the same performance with M-MMSE (for the considered setup) for any value of $N$ and $\kappa$. As $\kappa$ increases, both RZF and MRT provide better performance. This is further investigated for $N=64$ or 150 in Fig. 5. The performance gap between RZF and MRT reduces in both cases quite rapidly as $\kappa \leq 20$; this is because, on average, the intra-cell interference reduces when $\kappa$ increases. However, the gap reduces much more slowly for $40 \leq \kappa \leq 100$.

\section{Conclusions}

We investigated the effect of uncorrelated Rician fading channels on the UL and DL ergodic achievable rates of MRT/MRC and RZF/S-MMSE in Massive MIMO under the assumption of channel estimation errors and pilot contamination. Recent results from random matrix theory were used to find asymptotic approximations for S-MMSE/RZF that depend only on the long-term channel statistics, the Rician factors and the deterministic components. Numerical results indicated that these approximations are asymptotically tight, but also accurate for systems with finite dimensions. Applied to practical networks, such results can be used to simulate the network behavior without to carry out extensive MonteCarlo simulations and get important insights into the system behavior, with respect to the LoS vectors, CSI quality and induced interference. For a simplified channel model with orthogonal LoS components across UEs, we analytically evaluated the impact of the Rician factor of each UE on both the residual interference, induced by channel estimations errors, and pilot contamination. Also, we determined numerically how the number of antennas can be reduced for achieving a given target rate.

To make the problem analytically more tractable, singlecell processing and uncorrelated Rician fading channels were only considered in this work. Spurred by the new results in [5] (that disproved previous belief on the fundamental limits of Massive MIMO), an important follow-up of this work is to consider optimal M-MMSE combining/precoding and spatially correlated Rician fading channels. This latter case can potentially be addressed by extending the random matrix theory tools developed in [37] but applied to the DL of a single-cell multiuser large-scale MIMO network under spatially correlated Rician fading, with a common spatial channel correlation matrix among UEs.

\section{APPENDIX A \\ ASYMPTOTIC ANALYSIS OF MRT}

We start dividing the numerator and denominator of $\gamma_{j k}^{\mathrm{dl}}$ by $1 / N$. Then, we obtain

$\frac{\theta_{j} N\left|\mathbb{E}\left\{\frac{1}{N} \mathbf{h}_{j j k}^{H} \widehat{\mathbf{h}}_{j j k}\right\}\right|^{2}}{\frac{1}{N \rho_{\mathrm{d} l}}+\sum_{l=1}^{L} \sum_{i=1}^{K} \theta_{l} N \mathbb{E}\left\{\left|\frac{1}{N} \mathbf{h}_{l j k}^{H} \widehat{\mathbf{h}}_{l i}\right|^{2}\right\}-\theta_{j} N\left|\mathbb{E}\left\{\frac{1}{N} \mathbf{h}_{j j k}^{H} \widehat{\mathbf{h}}_{j j k}\right\}\right|^{2}}$. 
1) Signal Power: Using straightforward computations yields $\frac{1}{N} \mathbf{h}_{j j k}^{H} \widehat{\mathbf{h}}_{j j k} \asymp \phi_{j j k}+\frac{1}{N} \overline{\mathbf{h}}_{j j k}^{H} \overline{\mathbf{h}}_{j j k}$ and

$$
\theta_{j} N \asymp\left(\frac{1}{K} \sum_{k=1}^{K}\left(\phi_{j j k}+\frac{1}{N} \overline{\mathbf{h}}_{j j k}^{H} \overline{\mathbf{h}}_{j j k}\right)\right)^{-1} .
$$

Therefore, we have that

$$
\theta_{j} N\left|\mathbb{E}\left\{\frac{1}{N} \mathbf{h}_{j j k}^{H} \widehat{\mathbf{h}}_{j j k}\right\}\right|^{2} \asymp \bar{\theta}_{j}\left(\phi_{j j k}+\frac{1}{N} \overline{\mathbf{h}}_{j j k}^{H} \overline{\mathbf{h}}_{j j k}\right)^{2}
$$

with $\bar{\theta}_{j}=\left(\frac{1}{K} \sum_{k=1}^{K}\left(\phi_{j j k}+\frac{1}{N} \overline{\mathbf{h}}_{j j k}^{H} \overline{\mathbf{h}}_{j j k}\right)\right)^{-1}$.

2) Interference Power: We proceed computing the deterministic equivalent of the interference power. To begin with, we rewrite it as follows:

$$
\begin{aligned}
& \sum_{l=1}^{L} \sum_{i=1}^{K} \theta_{l} N \mathbb{E}\left\{\left|\frac{1}{N} \mathbf{h}_{l j k}^{H} \widehat{\mathbf{h}}_{l l i}\right|^{2}\right\}-\theta_{j} N\left|\mathbb{E}\left\{\frac{1}{N} \mathbf{h}_{j j k}^{H} \widehat{\mathbf{h}}_{j j k}\right\}\right|^{2} \\
& =s_{j k}^{(I)}+s_{j k}^{(P)}+\theta_{j} N \operatorname{var}\left[\frac{1}{N} \mathbf{h}_{j j k}^{H} \widehat{\mathbf{h}}_{j j k}\right]
\end{aligned}
$$

where $s_{j k}^{(I)}$ accounts for the intracell and intercell interference:

$$
s_{j k}^{(I)}=\sum_{l=1}^{L} \theta_{l} N \sum_{i=1, i \neq k}^{K} \mathbb{E}\left\{\left|\frac{1}{N} \mathbf{h}_{l j k}^{H} \widehat{\mathbf{h}}_{l l i}\right|^{2}\right\}
$$

and $s_{j k}^{(P)}$ is due to pilot contamination:

$$
s_{j k}^{(P)}=\sum_{l=1, l \neq j}^{L} \theta_{l} N \mathbb{E}\left\{\left|\frac{1}{N} \mathbf{h}_{l j k}^{H} \widehat{\mathbf{h}}_{l l k}\right|^{2}\right\} .
$$

We start computing an asymptotic expression for $s_{j k}^{(I)}$. To this end, we write $s_{j k}^{(I)}$ as follows:

$$
\begin{aligned}
s_{j k}^{(I)} & =\sum_{l=1, l \neq j}^{L} \theta_{l} N \sum_{i=1, i \neq k}^{K} \mathbb{E}\left\{\left|\frac{1}{N} \mathbf{h}_{l j k}^{H} \widehat{\mathbf{h}}_{l l i}\right|^{2}\right\} \\
& +\theta_{j} N \sum_{i=1, i \neq k}^{K} \mathbb{E}\left\{\left|\frac{1}{N} \mathbf{h}_{j j k}^{H} \widehat{\mathbf{h}}_{j j i}\right|^{2}\right\} .
\end{aligned}
$$

Consider the first term in (73). Since $\widehat{\mathbf{h}}_{l l i}$ in (4) is independent from $\mathbf{h}_{l j k}$ and $\mathbb{E}\left[\mathbf{h}_{l j k}^{H} \mathbf{h}_{l j k}\right]=d_{l j k}$, it easily follows that:

$$
\begin{aligned}
\frac{1}{N^{2}}\left|\mathbf{h}_{l j k}^{H} \widehat{\mathbf{h}}_{l l i}\right|^{2} & \asymp \frac{1}{N^{2}} \operatorname{tr}\left(d_{l j k}\left(\phi_{l l i} \mathbf{I}_{N}+\overline{\mathbf{h}}_{l l i} \overline{\mathbf{h}}_{l l i}^{H}\right)\right) \\
& \asymp \frac{1}{N} d_{l j k}\left(\phi_{l l i}+\frac{1}{N} \overline{\mathbf{h}}_{l i i} \overline{\mathbf{h}}_{l l i}^{H}\right) .
\end{aligned}
$$

Therefore, we have that:

$$
\begin{aligned}
& \sum_{l=1, l \neq j}^{L} \theta_{l} N \sum_{i=1, i \neq k}^{K} \mathbb{E}\left\{\left|\frac{1}{N} \mathbf{h}_{l j k}^{H} \widehat{\mathbf{h}}_{l l i}\right|^{2}\right\} \\
& \asymp \frac{1}{N} \sum_{l=1, l \neq j}^{L} \sum_{i=1, i \neq k}^{K} \bar{\theta}_{l} d_{l j k}\left(\phi_{l l i}+\frac{1}{N} \overline{\mathbf{h}}_{l l i} \overline{\mathbf{h}}_{l l i}^{H}\right) .
\end{aligned}
$$

Consider the second term in (73) and observe that

$$
\begin{aligned}
& \left|\frac{1}{N} \mathbf{h}_{j j k}^{H} \widehat{\mathbf{h}}_{j j i}\right|^{2} \\
& \asymp \frac{1}{N}\left(d_{j j k} \frac{1}{N} \widehat{\mathbf{h}}_{j j i}^{H} \widehat{\mathbf{h}}_{j j i}+\frac{1}{N} \overline{\mathbf{h}}_{j j k}^{H} \widehat{\mathbf{h}}_{j j i} \widehat{\mathbf{h}}_{j j i}^{H} \overline{\mathbf{h}}_{j j k}\right) \\
& \asymp \frac{1}{N} d_{j j k}\left(\phi_{j j i}+\frac{1}{N} \overline{\mathbf{h}}_{j j i} \overline{\mathbf{h}}_{j j i}^{H}\right) \\
& \quad+\frac{1}{N}\left(\frac{1}{N} \overline{\mathbf{h}}_{j j k}^{H}\left(\phi_{j j i} \mathbf{I}_{N}+\overline{\mathbf{h}}_{j j i} \overline{\mathbf{h}}_{j j i}^{H}\right) \overline{\mathbf{h}}_{j j k}\right) \\
& \asymp \frac{1}{N} d_{j j k}\left(\phi_{j j i}+\frac{1}{N} \overline{\mathbf{h}}_{j j i} \overline{\mathbf{h}}_{j j i}^{H}\right) \\
& \quad+\frac{1}{N}\left(\frac{1}{N} \phi_{j j i} \overline{\mathbf{h}}_{j j k}^{H} \overline{\mathbf{h}}_{j j k}\right)+\frac{1}{N}\left(\frac{1}{N}\left|\overline{\mathbf{h}}_{j j i}^{H} \overline{\mathbf{h}}_{j j k}\right|^{2}\right) .
\end{aligned}
$$

From the above results, it follows that

$$
\begin{gathered}
\theta_{j} N \sum_{i=1, i \neq k}^{K} \mathbb{E}\left\{\left|\frac{1}{N} \mathbf{h}_{j j k}^{H} \widehat{\mathbf{h}}_{j j i}\right|^{2}\right\} \asymp \frac{1}{N} \bar{\theta}_{j} \sum_{i=1, i \neq k}^{K} d_{j j k}\left(\phi_{j j i}+\frac{1}{N} \overline{\mathbf{h}}_{j j i} \overline{\mathbf{h}}_{j j i}^{H}\right) \\
+\frac{1}{N} \phi_{j j i} \overline{\mathbf{h}}_{j j k}^{H} \overline{\mathbf{h}}_{j j k}+\frac{1}{N}\left|\overline{\mathbf{h}}_{j j i}^{H} \overline{\mathbf{h}}_{j j k}\right|^{2} .
\end{gathered}
$$

Putting (75) and (80) together yields:

$$
\begin{aligned}
s_{j k}^{(I)} \asymp & \frac{1}{N} \sum_{l=1}^{L} \sum_{i=1, i \neq k}^{K} \bar{\theta}_{l} d_{l j k}\left(\phi_{l l i}+\frac{1}{N} \overline{\mathbf{h}}_{l l i} \overline{\mathbf{h}}_{l l i}^{H}\right) \\
& +\frac{1}{N^{2}} \bar{\theta}_{j} \sum_{i=1, i \neq k}^{K} \phi_{j j i} \overline{\mathbf{h}}_{j j k}^{H} \overline{\mathbf{h}}_{j j k}+\left|\overline{\mathbf{h}}_{j j i}^{H} \overline{\mathbf{h}}_{j j k}\right|^{2}
\end{aligned}
$$

We now proceed considering the pilot contamination term $s_{j k}^{(P)}$. Since $\widehat{\mathbf{h}}_{l l k}$ depends on $\mathbf{h}_{l j k}$, we must proceed as follows. Recall that $\widehat{\mathbf{h}}_{l l k}=\overline{\mathbf{h}}_{l l k}+\alpha_{l l k}\left(\mathbf{y}_{l k}^{\text {tr }}-\overline{\mathbf{h}}_{j j k}\right)$ and $\alpha_{l l k}=$ $d_{l l k}\left(\frac{1}{\rho^{\mathrm{tr}}}+\sum_{n=1}^{L} d_{l n k}\right)^{-1}$. Rewrite $\widehat{\mathbf{h}}_{l l k}$ as follows

$$
\begin{aligned}
\widehat{\mathbf{h}}_{l l k} & =\overline{\mathbf{h}}_{l l k}+\alpha_{l l k}\left(\mathbf{y}_{l k}^{\mathrm{tr}}-\mathbf{h}_{l j k}+\mathbf{h}_{l j k}-\overline{\mathbf{h}}_{j j k}\right) \\
& =\widehat{\widehat{\mathbf{h}}}_{l l k}+\alpha_{l l k} \mathbf{h}_{l j k}
\end{aligned}
$$

with $\widehat{\hat{\mathbf{h}}}_{l l k}=\overline{\mathbf{h}}_{l l k}+\alpha_{l l k}\left(\mathbf{y}_{j k}^{\mathrm{tr}}-\mathbf{h}_{l j k}-\overline{\mathbf{h}}_{j j k}\right)=\widehat{\mathbf{h}}_{l l k}-$ $\alpha_{l l k} \mathbf{h}_{l j k}$. Using (82) we may write

$$
\begin{aligned}
\frac{1}{N^{2}}\left|\mathbf{h}_{l j k}^{H} \widehat{\mathbf{h}}_{l l k}\right|^{2} & =\alpha_{l l k}^{2}\left|\frac{1}{N} \mathbf{h}_{l j k}^{H} \mathbf{h}_{l j k}\right|^{2}+\left|\frac{1}{N} \mathbf{h}_{l j k}^{H} \widehat{\widehat{\mathbf{h}}}_{l l k}\right|^{2} \\
& +2 \alpha_{l l k} \Re e\left\{\frac{1}{N^{2}} \widehat{\widehat{\mathbf{h}}}_{l l k} \mathbf{h}_{l j k} \mathbf{h}_{l j k}^{H} \mathbf{h}_{l j k}\right\} .
\end{aligned}
$$

Observe that $\alpha_{l l k}^{2}\left|\frac{1}{N} \mathbf{h}_{l j k}^{H} \mathbf{h}_{l j k}\right|^{2} \asymp \alpha_{l l k}^{2} d_{l j k}^{2}=\phi_{l j k}^{2}$. Since $\widehat{\widehat{\mathbf{h}}}_{l l k}$ is independent of $\mathbf{h}_{l j k}$, we have that $\left|\frac{1}{N} \mathbf{h}_{l j k}^{H} \widehat{\widehat{\mathbf{h}}}_{l l k}\right|^{2} \asymp$ $\frac{1}{N} d_{l j k}\left(\frac{1}{N} \widehat{\widehat{\mathbf{h}}}_{l l k}^{H} \widehat{\widehat{\mathbf{h}}}_{l l k}\right)$ with

$$
\begin{aligned}
\frac{1}{N} \widehat{\widehat{\mathbf{h}}}_{l l k}^{H} \widehat{\widehat{\mathbf{h}}}_{l l k} & =\frac{1}{N} \widehat{\mathbf{h}}_{l l k}^{H} \widehat{\mathbf{h}}_{l l k}-\alpha_{l l k} \frac{1}{N} \mathbf{h}_{l j k}^{H} \widehat{\mathbf{h}}_{l l k} \\
& -\alpha_{l l k} \frac{1}{N} \widehat{\mathbf{h}}_{l l k}^{H} \mathbf{h}_{l j k}+\alpha_{l l k}^{2} \frac{1}{N} \mathbf{h}_{l j k}^{H} \mathbf{h}_{l j k} .
\end{aligned}
$$

Observe now that $\frac{1}{N} \widehat{\mathbf{h}}_{l l k}^{H} \widehat{\mathbf{h}}_{l l k} \asymp \phi_{l l k}+\frac{1}{N} \overline{\mathbf{h}}_{l l k}^{H} \overline{\mathbf{h}}_{l l k}$ whereas

$$
\frac{1}{N} \mathbf{h}_{l j k}^{H} \widehat{\mathbf{h}}_{l l k} \asymp \alpha_{l l k} d_{l j k}
$$




$$
\gamma_{j k}^{\mathrm{ul}}=\frac{\left|\frac{1}{N} \widehat{\mathbf{h}}_{j j k}^{H} \mathbf{Q}_{j} \widehat{\mathbf{h}}_{j j k}\right|^{2}}{\mathbb{E}\left\{\sum_{l=1, l \neq j}^{L} \sum_{i=1}^{K}\left|\frac{1}{N} \widehat{\mathbf{h}}_{j j k}^{H} \mathbf{Q}_{j} \mathbf{h}_{j l i}\right|^{2}+\sum_{i=1, i \neq k}^{K}\left|\frac{1}{N} \widehat{\mathbf{h}}_{j j k}^{H} \mathbf{Q}_{j} \mathbf{h}_{j j i}\right|^{2}+\left|\frac{1}{N} \widehat{\mathbf{h}}_{j j k}^{H} \mathbf{Q}_{j} \mathbf{e}_{j j k}\right|^{2}+\frac{1}{\rho^{\mathrm{ul}}} \frac{1}{N} \widehat{\mathbf{h}}_{j j k}^{H} \mathbf{Q}_{j} \widehat{\mathbf{h}}_{j j k} \mid \widehat{\mathbf{H}}_{j j}\right\}}
$$

and $\frac{1}{N} \mathbf{h}_{l j k}^{H} \mathbf{h}_{l j k} \asymp d_{l j k}$. Putting all the above results together yields

$s_{j k}^{(P)} \asymp \sum_{l=1, l \neq j}^{L} \bar{\theta}_{l} \phi_{l j k}^{2}+\frac{1}{N} \sum_{l=1, l \neq j}^{L} \bar{\theta}_{l} d_{l j k}\left(\phi_{l l k}+\frac{1}{N} \overline{\mathbf{h}}_{l l k} \overline{\mathbf{h}}_{l l k}^{H}\right)$

where we have neglected the term $\alpha_{l l k} d_{l j k}^{2}$, which appears only $L-1$ times. Combining the above results together yields

$s_{j k}^{(I)}+s_{j k}^{(P)} \asymp \frac{1}{N} \sum_{l=1}^{L} \sum_{i=1}^{K} \bar{\theta}_{l} d_{l j k}\left(\phi_{l l i}+\frac{1}{N} \overline{\mathbf{h}}_{l l i} \overline{\mathbf{h}}_{l l i}^{H}\right)$

$+\frac{1}{N^{2}} \bar{\theta}_{j} \sum_{i=1, i \neq k}^{K}\left(\phi_{j j i} \overline{\mathbf{h}}_{j j k}^{H} \overline{\mathbf{h}}_{j j k}+\left|\overline{\mathbf{h}}_{j j i}^{H} \overline{\mathbf{h}}_{j j k}\right|^{2}\right)+\sum_{l \neq j} \bar{\theta}_{l} \phi_{l j k}^{2}$

where we have added $\frac{1}{N} \bar{\theta}_{j} d_{j j k}\left(\phi_{j j k}+\frac{1}{N} \overline{\mathbf{h}}_{j j k} \overline{\mathbf{h}}_{j j k}^{H}\right)$, which is negligible for large $N$. We are only left with $\operatorname{var}\left[\frac{1}{N} \mathbf{h}_{j j k}^{H} \widehat{\mathbf{h}}_{j j k}\right]$, which can be rewritten as:

$\operatorname{var}\left[\frac{1}{N} \mathbf{h}_{j j k}^{H} \widehat{\mathbf{h}}_{j j k}\right]=\mathbb{E}\left\{\left|\frac{1}{N} \mathbf{h}_{j j k}^{H} \widehat{\mathbf{h}}_{j j k}\right|^{2}\right\}-\left|\mathbb{E}\left\{\frac{1}{N} \mathbf{h}_{j j k}^{H} \widehat{\mathbf{h}}_{j j k}\right\}\right|^{2}$

from which it is easily follows that $\operatorname{var}\left[\frac{1}{N} \mathbf{h}_{j j k}^{H} \widehat{\mathbf{h}}_{j j k}\right] \asymp 0$ since $\frac{1}{N} \mathbf{h}_{j j k}^{H} \widehat{\mathbf{h}}_{j j k} \asymp \phi_{j j k}+\frac{1}{N} \overline{\mathbf{h}}_{j j k}^{H} \overline{\mathbf{h}}_{j j k}$.

\section{APPENDIX B}

\section{ASYMPTOTIC ANALYSIS OF S-MMSE}

We start by dividing the numerator and denominator of $\gamma_{j k}^{\mathrm{ul}}$ by $1 / N$. Then, we obtain (79).

B.1) Preliminaries: To begin with, we define $\mathbf{Q}_{j k}$ as:

$$
\mathbf{Q}_{j k}=\left(\frac{1}{N} \sum_{i \neq k} \widehat{\mathbf{h}}_{j j i} \widehat{\mathbf{h}}_{j j i}^{H}+\lambda_{j} \mathbf{I}_{N}\right)^{-1} .
$$

Then, the following relations hold true:

$$
\begin{aligned}
{\left[\tilde{\mathbf{Q}}_{j}\right]_{k k} } & =\frac{1}{\lambda\left(1+\widehat{\mathbf{h}}_{j j k}^{H} \mathbf{Q}_{j} \widehat{\mathbf{h}}_{j j k}\right)} \\
\mathbf{Q}_{j} & =\mathbf{Q}_{j k}-\lambda_{j}\left[\tilde{\mathbf{Q}}_{j}\right]_{k k} \mathbf{Q}_{j k} \frac{1}{N} \widehat{\mathbf{h}}_{j j k} \widehat{\mathbf{h}}_{j j k}^{H} \mathbf{Q}_{j k} \\
\mathbf{Q}_{j} \widehat{\mathbf{h}}_{j j k} & =\frac{\mathbf{Q}_{j k} \widehat{\mathbf{h}}_{j j k}}{1+\widehat{\mathbf{h}}_{j j k}^{H} \mathbf{Q}_{j} \widehat{\mathbf{h}}_{j j k}}=\lambda_{j}\left[\tilde{\mathbf{Q}}_{j}\right]_{k k} \mathbf{Q}_{j k} \widehat{\mathbf{h}}_{j j k} .
\end{aligned}
$$

These relations will be extensively used in the asymptotic calculations of the SINR, where the replacement by $\mathbf{Q}_{j k}$ allows to ensure the dependence of the resolvent matrix from $\widehat{\mathbf{h}}_{j j k}$. However, the direct replacement of the deterministic equivalents associated with $\mathbf{Q}_{j k}$ by those of $\mathbf{Q}_{j}$ cannot be performed in all cases - since we are dealing with non-centered random variables - especially when quadratic forms are involved. Some technical derivations are required to express all the terms in terms of only the deterministic equivalents of $\mathbf{Q}_{j}$. Due to space limitations, we cannot include all the technical derivations; we refer the interested reader to the extended version [39].

B.2) Signal power: Applying the matrix inversion lemma we may write:

$$
\frac{1}{N} \widehat{\mathbf{h}}_{j j k}^{H} \mathbf{Q}_{j} \widehat{\mathbf{h}}_{j j k}=\lambda_{j}\left[\tilde{\mathbf{Q}}_{j}\right]_{k k} \frac{1}{N} \widehat{\mathbf{h}}_{j j k}^{H} \mathbf{Q}_{j k} \widehat{\mathbf{h}}_{j j k}
$$

From (94), $\frac{1}{N} \widehat{\mathbf{h}}_{j j k} \mathbf{Q}_{j k} \widehat{\mathbf{h}}_{j j k}=\frac{1}{\lambda_{j}\left[\widehat{\mathbf{Q}}_{j}\right]_{k k}}-1$. Using the fact that $\left[\tilde{\mathbf{Q}}_{j}\right]_{k k} \asymp\left[\widetilde{\mathbf{T}}_{j}\right]_{k k}$, we ultimately obtain:

$$
\frac{1}{N} \widehat{\mathbf{h}}_{j j k}^{H} \mathbf{Q}_{j} \widehat{\mathbf{h}}_{j j k} \asymp\left(1-\lambda_{j}\left[\widetilde{\mathbf{T}}_{j}\right]_{k k}\right) .
$$

and from the continuous mapping Theorem [12], $\left|\frac{1}{N} \widehat{\mathbf{h}}_{j j k}^{H} \mathbf{Q}_{j} \widehat{\mathbf{h}}_{j j k}\right|^{2} \asymp\left(1-\lambda_{j}\left[\widetilde{\mathbf{T}}_{j}\right]_{k k}\right)^{2}$.

B.3) Interference power: In the asymptotic regime, the conditional expectation with respect to $\widehat{\mathbf{H}}_{j j}$ can be replaced by the expectation. We thus consider computing the following equivalent interference $s_{j k}=s_{j k}^{(I)}+s_{j k}^{(P)}+o(1)$ where

$$
s_{j k}^{(I)}=\sum_{l=1}^{L} \sum_{i \neq k} \mathbb{E}\left\{\left|\frac{1}{N} \widehat{\mathbf{h}}_{j j k}^{H} \mathbf{Q}_{j} \mathbf{h}_{j l i}\right|^{2}\right\}
$$

accounts for the intracell and intercell interference and

$$
s_{j k}^{(P)}=\sum_{l \neq j} \mathbb{E}\left\{\left|\frac{1}{N} \widehat{\mathbf{h}}_{j j k}^{H} \mathbf{Q}_{j} \mathbf{h}_{j l k}\right|^{2}\right\}
$$

is due to pilot-sharing UEs. Let's start with $s_{j k}^{(P)}$ for which it easily follows that:

$$
s_{j k}^{(P)}=\sum_{l \neq j}\left(\phi_{j l k} \delta_{j} \lambda_{j}\left[\widetilde{\mathbf{T}}_{j}\right]_{k k}\right)^{2}+o(1) .
$$

The term $s_{j k}^{(I)}$ is decomposed as

$$
\begin{aligned}
s_{j k}^{(I)} & =\sum_{l \neq j} \sum_{i \neq k} \mathbb{E}\left\{\left|\frac{1}{N} \widehat{\mathbf{h}}_{j j k}^{H} \mathbf{Q}_{j} \mathbf{h}_{j l i}\right|^{2}\right\}+\sum_{i \neq k} \mathbb{E}\left\{\left|\frac{1}{N} \widehat{\mathbf{h}}_{j j k}^{H} \mathbf{Q}_{j} \mathbf{h}_{j j i}\right|^{2}\right\} \\
& \triangleq s_{j k, \text { outer }}^{(I)}+s_{j k, \text { inner }}^{(I)}
\end{aligned}
$$

where $s_{j k \text {,outer }}^{(I)}$ and $s_{j k, \text { inner }}^{(I)}$ represent the inter-cell and intracell interference, respectively. 


$$
\begin{aligned}
\lambda_{j}^{2}\left[\widetilde{\mathbf{T}}_{j}\right]_{k k}^{2} \sum_{i \neq k} \mathbb{E}\left\{\left|\frac{1}{N} \overline{\mathbf{h}}_{j j k}^{H} \mathbf{Q}_{j k} \mathbf{h}_{j l i}\right|^{2}\right\} & =\frac{1}{\left(1+\delta_{j} \phi_{j j k}\right)^{2}} \sum_{i \neq k} \mathbb{E}\left\{\left|\frac{1}{N} \overline{\mathbf{h}}_{j j k}^{H} \mathbf{Q}_{j} \mathbf{h}_{j l i}\right|^{2}\right\} \\
& -\phi_{j j k}\left(\frac{\frac{1}{N} \overline{\mathbf{h}}_{j j k}^{H} \mathbf{T}_{j} \overline{\mathbf{h}}_{j j k}}{\left(1+\delta_{j} \phi_{j j k}\right)^{2}}\right)^{2} \sum_{i \neq k} \frac{1}{N^{2}} \mathbb{E}\left\{\mathbf{h}_{j l i}^{H} \mathbf{Q}_{j k}^{2} \mathbf{h}_{j l i}\right\}+o(1) .
\end{aligned}
$$

$$
\begin{aligned}
& s_{j k, \text { outer }}^{(I)}=\sum_{l \neq j} \sum_{i \neq k} \frac{\mathbb{E}\left\{\left|\frac{1}{N} \overline{\mathbf{h}}_{j j k}^{H} \mathbf{Q}_{j} \mathbf{h}_{j l i}\right|^{2}\right\}}{\left(1+\delta_{j} \phi_{j j k}\right)^{2}}+\phi_{j j k}\left(\lambda_{j}^{2}\left[\widetilde{\mathbf{T}}_{j}\right]_{k k}^{2}-\left(\frac{\left.\left.\frac{1}{N} \overline{\mathbf{h}}_{j j k}^{H} \mathbf{T}_{j} \overline{\mathbf{h}}_{j j k}\right){ }^{2}\right) \sum_{i \neq k} \frac{1}{N^{2}} \mathbb{E}\left\{\mathbf{h}_{j l i}^{H} \mathbf{Q}_{j}^{2} \mathbf{h}_{j l i}\right\}+o(1)}{s_{j k, \text { inner }}}=\sum_{i \neq k} \frac{\mathbb{E}\left\{\left|\frac{1}{N} \overline{\mathbf{h}}_{j j k}^{H} \mathbf{Q}_{j} \mathbf{h}_{j j i}\right|^{2}\right\}}{\left(1+\delta_{j} \phi_{j j k}\right)^{2}}+\phi_{j j k}\left(\lambda_{j}^{2}\left[\widetilde{\mathbf{T}}_{j}\right]_{k k}^{2}-\left(\frac{\frac{1}{N} \overline{\mathbf{h}}_{j j k}^{H} \mathbf{T}_{j} \overline{\mathbf{h}}_{j j k}}{\left(1+\delta_{j} \phi_{j j k}\right)^{2}}\right)^{2}\right) \sum_{i \neq k} \frac{1}{N^{2}} \mathbb{E}\left\{\mathbf{h}_{j j i}^{H} \mathbf{Q}_{j}^{2} \mathbf{h}_{j j i}\right\}+o(1) .\right.\right.
\end{aligned}
$$

$$
\begin{aligned}
& \phi_{j j k}\left(\lambda_{j}^{2}\left[\widetilde{\mathbf{T}}_{j}\right]_{k k}^{2}-\left(\frac{\frac{1}{N} \overline{\mathbf{h}}_{j j k}^{H} \mathbf{T}_{j} \overline{\mathbf{h}}_{j j k}}{\left(1+\delta_{j} \phi_{j j k}\right)^{2}}\right)^{2}\right) \sum_{i \neq k} \frac{1}{N^{2}} \mathbb{E}\left\{\mathbf{h}_{j l i}^{H} \mathbf{Q}_{j}^{2} \mathbf{h}_{j l i}\right\}=\left(\lambda_{j}^{2}\left[\widetilde{\mathbf{T}}_{j} \boldsymbol{\Phi}_{j j} \widetilde{\mathbf{T}}_{j}\right]_{k k}-\tilde{\beta}_{j j k}\right) \frac{1}{N} \sum_{i=1}^{K} \frac{1-F_{j}}{\Delta_{j}} \gamma_{j l i} \\
& +\left(\lambda_{j}^{2}\left[\widetilde{\mathbf{T}}_{j} \boldsymbol{\Phi}_{j j} \widetilde{\mathbf{T}}_{j}\right]_{k k}-\tilde{\beta}_{j j k}\right)\left(\frac{1}{N} \sum_{i=1}^{K} \mu_{j l i} \bar{\nu}_{j}\right)+o(1) .
\end{aligned}
$$

We start with $s_{j k, \text { outer }}^{(I)}$. By using $\mathbf{Q}_{j} \widehat{\mathbf{h}}_{j j k}=$ $\lambda_{j}\left[\tilde{\mathbf{Q}}_{j}\right]_{k k} \mathbf{Q}_{j k} \widehat{\mathbf{h}}_{j j k}$ and taking the expectation over $\widehat{\mathbf{h}}_{j j k}$ yields

$$
\begin{aligned}
& s_{j k, \text { outer }}^{(I)}=\lambda_{j}^{2}\left[\widetilde{\mathbf{T}}_{j}\right]_{k k}^{2} \sum_{l \neq j} \sum_{i \neq k} \mathbb{E}\left\{\left|\frac{1}{N} \overline{\mathbf{h}}_{j j k}^{H} \mathbf{Q}_{j k} \mathbf{h}_{j l i}\right|^{2}\right\} \\
& +\lambda_{j}^{2} \phi_{j j k}\left[\widetilde{\mathbf{T}}_{j}\right]_{k k}^{2} \sum_{l \neq j} \sum_{i \neq k} \mathbb{E}\left\{\frac{1}{N^{2}} \mathbf{h}_{j l i}^{H} \mathbf{Q}_{j k}^{2} \mathbf{h}_{j l i}\right\}+o(1) .
\end{aligned}
$$

In order to get simplified expressions, we need to work with $\mathbf{Q}_{j}$ instead of $\mathbf{Q}_{j k}$. As mentioned before, a direct replacement of $\mathbf{Q}_{j}$ with $\mathbf{Q}_{j k}$ is not allowed since it induces a non-vanishing error that needs to be evaluated beforehand. Hence, we use the following identity

$$
\mathbf{Q}_{j}=\mathbf{Q}_{j k}-\lambda_{j}\left[\tilde{\mathbf{Q}}_{j}\right]_{k k} \mathbf{Q}_{j k} \frac{1}{N} \widehat{\mathbf{h}}_{j j k} \widehat{\mathbf{h}}_{j j k}^{H} \mathbf{Q}_{j k}
$$

to obtain

$$
\begin{aligned}
& \sum_{i \neq k} \mathbb{E}\left\{\left|\frac{1}{N} \overline{\mathbf{h}}_{j j k}^{H} \mathbf{Q}_{j} \mathbf{h}_{j l i}\right|^{2}\right\}=\sum_{i \neq k} \mathbb{E}\left\{\left|\frac{1}{N} \overline{\mathbf{h}}_{j j k}^{H} \mathbf{Q}_{j k} \mathbf{h}_{j l i}\right|^{2}\right\} \\
& +\sum_{i \neq k} \lambda_{j}^{2}\left[\widetilde{\mathbf{T}}_{j}\right]_{k k}^{2} \frac{1}{N^{4}} \mathbb{E}\left\{\left|\overline{\mathbf{h}}_{j j k}^{H} \mathbf{Q}_{j k} \widehat{\mathbf{h}}_{j j k}\right|^{2}\left|\mathbf{h}_{j l i}^{H} \mathbf{Q}_{j k} \widehat{\mathbf{h}}_{j j k}\right|^{2}\right\} \\
& -\sum_{i \neq k} \lambda_{j}\left[\widetilde{\mathbf{T}}_{j}\right]_{k k} \frac{1}{N^{3}} \mathbb{E}\left\{\overline{\mathbf{h}}_{j j k}^{H} \mathbf{Q}_{j k} \widehat{\mathbf{h}}_{j j k} \widehat{\mathbf{h}}_{j j k}^{H} \mathbf{Q}_{j k} \mathbf{h}_{j l i} \widehat{\mathbf{h}}_{j l i}^{H} \mathbf{Q}_{j k} \overline{\mathbf{h}}_{j j k}\right\} \\
& -\sum_{i \neq k} \lambda_{j}\left[\widetilde{\mathbf{T}}_{j}\right]_{k k} \frac{1}{N^{3}} \mathbb{E}\left\{\overline{\mathbf{h}}_{j j k}^{H} \mathbf{Q}_{j k} \mathbf{h}_{j l i} \mathbf{h}_{j l i}^{H} \mathbf{Q}_{j k} \widehat{\mathbf{h}}_{j j k} \widehat{\mathbf{h}}_{j j k}^{H} \mathbf{Q}_{j k} \overline{\mathbf{h}}_{j j k}\right\} \\
& +o(1)
\end{aligned}
$$

from which (89) follows (details are omitted and can be found in [39]). By plugging (89) into (90) and replacing $\mathbf{Q}_{j k}$ with $\mathbf{Q}_{j}$ in the second term (up to a vanishing error), we obtain (90) on the top of this page. Similarly, we can show that $s_{j k \text {,inner }}^{(I)}$ is given by 91 .

Consider the term $\sum_{i \neq k} \mathbb{E}\left\{\left|\frac{1}{N} \overline{\mathbf{h}}_{j j k}^{H} \mathbf{Q}_{j} \mathbf{h}_{j l i}\right|\right\}^{2}$ in (90) for $l \neq j$. By using the identity

$$
\mathbf{Q}_{j}=\mathbf{Q}_{j i}-\lambda_{j}\left[\tilde{\mathbf{Q}}_{j}\right]_{i i} \frac{1}{N} \mathbf{Q}_{j i} \widehat{\mathbf{h}}_{j j i} \widehat{\mathbf{h}}_{j j i}^{H} \mathbf{Q}_{j i}
$$

we obtain (after some calculus reported in [39])

$$
\begin{aligned}
& \sum_{i \neq k} \mathbb{E}\left\{\left|\frac{1}{N} \overline{\mathbf{h}}_{j j k}^{H} \mathbf{Q}_{j} \mathbf{h}_{j l i}\right|^{2}\right\}=\lambda_{j}^{2}\left[\widetilde{\mathbf{T}}_{j} \operatorname{diag}\left\{\phi_{j l i}^{2} \delta_{j}^{2}\right\} \widetilde{\mathbf{T}}_{j}\right]_{k k} \\
& -\lambda_{j}^{2}\left[\widetilde{\mathbf{T}}_{j}\right]_{k k}^{2} \delta_{j}^{2} \phi_{j l k}^{2}+\tilde{\beta}_{j j k} \frac{1-F_{j}}{\Delta_{j}} \frac{1}{N} \sum_{i=1}^{K} \gamma_{j l i} \\
& +\gamma_{j j k} \lambda_{j}^{2} \frac{\widetilde{\vartheta}_{j}}{\Delta_{j}} \frac{1}{N} \sum_{i=1}^{K} \gamma_{j l i} \\
& +\left(\frac{1-F_{j}}{\Delta_{j}} \gamma_{j j k}+\bar{\nu}_{j} \tilde{\beta}_{j j k}\right)\left(\frac{1}{N} \sum_{i=1}^{K} \mu_{j l i}\right)+o(1)
\end{aligned}
$$

where $\gamma_{j l i}$ is defined in (41), $\widetilde{\vartheta}_{j}=\frac{1}{N} \operatorname{tr}\left(\boldsymbol{\Phi}_{j j} \widetilde{\mathbf{T}}_{j}\right)^{2}$ and

$$
\begin{aligned}
\tilde{\beta}_{j j k} & =\lambda_{j}^{2}\left[\widetilde{\mathbf{T}}_{j} \boldsymbol{\Phi}_{j j} \widetilde{\mathbf{T}}_{j}\right]_{k k}-\lambda_{j}^{2} \phi_{j j k}\left[\widetilde{\mathbf{T}}_{j}\right]_{k k}^{2} \\
& +\phi_{j j k} \frac{\left|\frac{1}{N} \overline{\mathbf{h}}_{j j k}^{H} \mathbf{T}_{j} \overline{\mathbf{h}}_{j j k}\right|^{2}}{\left(1+\delta_{j} \phi_{j j k}\right)^{2}} .
\end{aligned}
$$

Consider now the second term $\frac{1}{N^{2}} \mathbb{E}\left\{\mathbf{h}_{j l i}^{H} \mathbf{Q}_{j}^{2} \mathbf{h}_{j l i}\right\}$ in (90). By using the results in [37], [39], it can be proved that:

$$
\begin{aligned}
\sum_{i \neq k} \frac{1}{N^{2}} \mathbb{E} & \left\{\mathbf{h}_{j l i}^{H} \mathbf{Q}_{j}^{2} \mathbf{h}_{j l i}\right\}=\frac{1}{N} \sum_{i=1}^{K} \mu_{j l i} \bar{\nu}_{j} \\
& +\lambda_{j}^{2}\left[\widetilde{\mathbf{T}}_{j} \boldsymbol{\Phi}_{j} \widetilde{\mathbf{T}}_{j}\right]_{i i} \phi_{j l i}^{2} \delta_{j}^{2} \bar{\nu}_{j} \frac{1-F_{j}}{\Delta_{j}} \gamma_{j l i}+o(1)
\end{aligned}
$$




$$
\begin{aligned}
\sum_{i \neq k} \mathbb{E}\left\{\left|\frac{1}{N} \overline{\mathbf{h}}_{j j k}^{H} \mathbf{Q}_{j} \mathbf{h}_{j j i}\right|^{2}\right\} & =\lambda_{j}^{2} \sum_{i \neq k}\left[\widetilde{\mathbf{T}}_{j}\right]_{i i}^{2} \mathbb{E}\left\{\left|\frac{1}{N} \overline{\mathbf{h}}_{j j k}^{H} \mathbf{Q}_{j i} \overline{\mathbf{h}}_{j j i}\right|^{2}\right\} \\
& +\frac{1}{N} \sum_{i \neq k}\left(d_{j j i}-\phi_{j j i}\left(1-\lambda_{j}^{2}\left[\widetilde{\mathbf{T}}_{j}\right]_{i i}^{2}\right)\right) \mathbb{E}\left\{\frac{1}{N} \overline{\mathbf{h}}_{j j k}^{H} \mathbf{Q}_{j}^{2} \overline{\mathbf{h}}_{j j k}\right\}+o(1) .
\end{aligned}
$$

$$
\begin{aligned}
& \sum_{i \neq k} \mathbb{E}\left|\frac{1}{N} \overline{\mathbf{h}}_{j j k}^{H} \mathbf{Q}_{j} \mathbf{h}_{j j i}\right|^{2}=\sum_{i \neq k} \frac{\mathbb{E}\left\{\left|\frac{1}{N} \overline{\mathbf{h}}_{j j k}^{H} \mathbf{Q}_{j} \overline{\mathbf{h}}_{j j i}\right|^{2}\right\}}{\left(1+\delta_{j} \phi_{j j i}\right)^{2}} \\
& +\frac{1}{N} \sum_{i \neq k}\left(d_{j j i}-\phi_{j j i}\left(1+\lambda_{j}^{2}\left[\widetilde{\mathbf{T}}_{j}\right]_{i i}^{2}-\frac{\left(\frac{1}{N} \overline{\mathbf{h}}_{j j i}^{H} \mathbf{T}_{j} \overline{\mathbf{h}}_{j j i}\right)^{2}}{\left(1+\delta_{j} \phi_{j j i}\right)^{4}}\right)\right) \mathbb{E}\left\{\frac{1}{N} \overline{\mathbf{h}}_{j j k}^{H} \mathbf{Q}_{j}^{2} \overline{\mathbf{h}}_{j j k}\right\}+o(1)
\end{aligned}
$$

$$
\begin{aligned}
& \phi_{j j k}\left(\lambda_{j}^{2}\left[\widetilde{\mathbf{T}}_{j}\right]_{k k}^{2}-\left(\frac{\frac{1}{N} \overline{\mathbf{h}}_{j j k}^{H} \mathbf{T}_{j} \overline{\mathbf{h}}_{j j k}}{\left(1+\delta_{j} \phi_{j j k}\right)^{2}}\right)^{2}\right) \sum_{i \neq k} \frac{1}{N^{2}} \mathbb{E}\left\{\mathbf{h}_{j j i}^{H} \mathbf{Q}_{j}^{2} \mathbf{h}_{j j i}\right\}= \\
&=\left(\lambda_{j}^{2}\left[\widetilde{\mathbf{T}}_{j} \boldsymbol{\Phi}_{j} \widetilde{\mathbf{T}}_{j}\right]_{k k}-\tilde{\beta}_{j j k}\right)\left(\frac{1-F_{j}}{\Delta_{j}} \frac{1}{N} \sum_{i=1}^{K} \bar{\nu}_{j} \gamma_{j j i}+\bar{\nu}_{j} \mu_{j j i}\right)+o(1) .
\end{aligned}
$$

from which (92) follows. By summing (110) and (92) for all $l \neq j$, we obtain:

$$
\begin{aligned}
& s_{j k, \text { outer }}^{(I)}=\sum_{l \neq j} \lambda_{j}^{2}\left[\widetilde{\mathbf{T}}_{j} \operatorname{diag}\left\{\phi_{j l i}^{2} \delta_{j}^{2}\right\} \widetilde{\mathbf{T}}_{j}\right]_{k k}-\lambda_{j}^{2}\left[\widetilde{\mathbf{T}}_{j}\right]_{k k}^{2} \delta_{j}^{2} \phi_{j l k}^{2} \\
& +\frac{1}{N} \sum_{i=1}^{K} \gamma_{j l i} \zeta_{j k}+\xi_{j k}\left(\frac{1}{N} \sum_{i=1}^{K} \mu_{j l i}\right)+o(1) .
\end{aligned}
$$

Let's consider now the term $s_{j k \text {,inner. We begin with }}^{(I)}$ $\sum_{i \neq k} \mathbb{E}\left\{\mid \frac{1}{N} \overline{\mathbf{h}}_{j j k}^{H} \mathbf{Q}_{j} \mathbf{h}_{j j i} \|^{2}\right\}$, which similar calculations allows to write explicitly as (106). The first term reduces to

$$
\begin{aligned}
& \lambda_{j}^{2} \sum_{i \neq k}\left[\widetilde{\mathbf{T}}_{j}\right]_{i i}^{2} \mathbb{E}\left\{\left|\frac{1}{N} \overline{\mathbf{h}}_{j j k}^{H} \mathbf{Q}_{j i} \overline{\mathbf{h}}_{j j i}\right|^{2}\right\}= \\
& =\sum_{i \neq k} \frac{\mathbb{E}\left\{\left|\frac{1}{N} \overline{\mathbf{h}}_{j j k}^{H} \mathbf{Q}_{j} \overline{\mathbf{h}}_{j j i}\right|^{2}\right\}}{\left(1+\delta_{j} \phi_{j j i}\right)^{2}} \\
& -\frac{1}{N} \sum_{i \neq k} \phi_{j j i} \frac{\left(\frac{1}{N} \overline{\mathbf{h}}_{j j i}^{H} \mathbf{T}_{j} \overline{\mathbf{h}}_{j j i}\right)^{2}}{\left(1+\delta_{j} \phi_{j j i}\right)^{4}} \mathbb{E}\left\{\frac{1}{N} \overline{\mathbf{h}}_{j j k}^{H} \mathbf{Q}_{j}^{2} \overline{\mathbf{h}}_{j j k}\right\}+o(1)
\end{aligned}
$$

such that 106 reduces to 107. Using standard calculations, we can show that:

$$
\begin{aligned}
& \sum_{i \neq k} \frac{\mathbb{E}\left\{\left|\frac{1}{N} \overline{\mathbf{h}}_{j j k}^{H} \mathbf{Q}_{j} \mathbf{h}_{j j i}\right|^{2}\right\}}{\left(1+\delta_{j} \phi_{j j i}\right)^{2}}=\left(\lambda_{j}^{2}\left[\widetilde{\mathbf{T}}_{j}^{2}\right]_{k k}-\lambda_{j}^{2}\left[\widetilde{\mathbf{T}}_{j}\right]_{k k}^{2}\right) \\
& +\tilde{\beta}_{j j k} \frac{1-F_{j}}{\Delta_{j}} \frac{1}{N} \sum_{i=1}^{K} \gamma_{j j i}+\gamma_{j j k} \frac{\lambda_{j}^{2} \widetilde{\vartheta}_{j}}{\Delta_{j}} \frac{1}{N} \sum_{i=1}^{K} \gamma_{j j i} \\
& +\left(\frac{1-F_{j}}{\Delta_{j}} \gamma_{j j k}+\bar{\nu}_{j} \beta_{j k}\right)\left(\frac{1}{N} \sum_{i=1}^{K} \mu_{j j i}\right)+o(1)
\end{aligned}
$$

and

$$
\begin{gathered}
\mathbb{E}\left\{\frac{1}{N} \mathbf{h}_{j j i}^{H} \mathbf{Q}_{j}^{2} \mathbf{h}_{j j i}\right\}=\frac{\vartheta_{j}}{\Delta_{j}}\left(d_{j j i}-\phi_{j j i}+\lambda_{j}^{2}\left[\widetilde{\mathbf{T}}_{j} \mathbf{\Phi}_{j} \widetilde{\mathbf{T}}_{j}\right]_{i i}\right) \\
\quad+\frac{1-F_{j}}{\Delta_{j}} \frac{\left[\widetilde{\mathbf{T}}_{j} \frac{1}{N} \overline{\mathbf{H}}_{j j}^{H} \overline{\mathbf{H}}_{j j} \widetilde{\mathbf{T}}_{j}\right]_{i i}}{\left(1+\tilde{\delta}_{j}\right)^{2}}+o(1)
\end{gathered}
$$

where $\vartheta_{j}=\frac{1}{N} \operatorname{tr}\left(\mathbf{T}_{j}^{2}\right)$. The second term in 91) can be approximated as in (108). Gathering all the above results together, we eventually obtain:

$$
\begin{aligned}
s_{j k, \text { inner }}^{(I)} & =\lambda_{j}^{2}\left(\left[\widetilde{\mathbf{T}}_{j}^{2}\right]_{k k}-\left[\widetilde{\mathbf{T}}_{j}\right]_{k k}^{2}\right)+\frac{1}{N} \sum_{i=1}^{K} \mu_{j j i} \xi_{j k} \\
& +\frac{1}{N} \sum_{i=1}^{K} \gamma_{j j i} \zeta_{j k}+o(1) .
\end{aligned}
$$

\section{APPENDIX C}

PROOF OF COROLlary 6

If the channel is modeled as in (53) and $\frac{1}{N} \overline{\mathbf{H}}_{j j}^{H} \overline{\mathbf{H}}_{j j}=$ $\frac{\kappa}{1+\kappa} \mathbf{I}_{K}$, then from (19) we obtain that

$$
\lambda_{j}=\lambda=\frac{K}{N}\left(\alpha(L-1)+\frac{1}{1+\kappa}-\phi\right)
$$

and $\boldsymbol{\Phi}_{j j}=\phi \mathbf{I}_{K}$ with $\phi_{j j k} \stackrel{(a)}{=} \phi=\frac{\nu}{(1+\kappa)^{2}}$, where $(a)$ follows from (54). Also, it turns out that $\delta_{j}=\delta$ and $\widetilde{\delta}_{j}=\widetilde{\delta}$ with

$$
\widetilde{\delta}=\phi \frac{K}{N}\left(\lambda+\lambda \widetilde{\delta}+\phi\left(1-\frac{K}{N}\right)+\frac{\kappa}{1+\kappa} \frac{1}{1+\widetilde{\delta}}\right)^{-1}
$$

where we have used that (after some simple calculus) $\phi \delta=$ $\widetilde{\delta}+\phi \frac{1-K / N}{\lambda}$. From the identity in 121 , it can be proved that $\widetilde{\delta}$ is the real positive solution, say $\widetilde{\delta}^{\star}$, of the following third order polynomial equation in 60 . 


$$
\begin{aligned}
\frac{\bar{s}}{\bar{\psi}} & =\left(\frac{d}{\phi}-1+\lambda^{2} \frac{N}{K \phi^{2}} \widetilde{\vartheta}\right)\left(-1+\frac{1}{\Delta}\right)-\frac{F(d-\phi)}{\phi \Delta}-\frac{F(L-1)}{\Delta}\left(\frac{\alpha}{\phi}-2 \lambda \alpha^{2}(1+\kappa)^{2} \delta^{\star} \frac{N}{K} \tilde{\delta}^{\star}\right) \\
& +(L-1)\left(\frac{\alpha}{\phi}-2 \lambda \alpha^{2}(1+\kappa)^{2} \delta^{\star} \frac{N}{K} \tilde{\delta}^{\star}+\alpha^{2}(1+\kappa)^{2}\left(\delta^{\star}\right)^{2} \lambda^{2} \frac{N}{K} \widetilde{\vartheta}\right)\left(-1+\frac{1}{\Delta}\right)
\end{aligned}
$$

$$
\begin{aligned}
\frac{\bar{s}}{\bar{\psi}} & =\left(\frac{1+\kappa-\nu}{\nu}+\lambda^{2}\left(\frac{N}{K}\right)^{2} \frac{\left(\tilde{\delta}^{\star}\right)^{2}}{\nu^{2}}(1+\kappa)^{4}\right)\left(-1+\frac{1}{\Delta}\right)-\frac{1+\kappa-\nu}{\nu^{2}} \frac{\kappa(1+\kappa)}{\Delta} \frac{N}{K} \frac{\left(\tilde{\delta}^{\star}\right)^{2}}{\left(1+\tilde{\delta}^{\star}\right)^{2}} \\
& +(L-1)\left(\frac{\alpha(1+\kappa)^{2}}{\nu}-2 \lambda \alpha^{2}(1+\kappa)^{2} \delta^{\star} \tilde{\delta}^{\star} \frac{N}{K}+\alpha^{2}(1+\kappa)^{2}\left(\delta^{\star}\right)^{2} \lambda^{2}\left(\frac{N}{K}\right)^{2}\left(\tilde{\delta}^{\star}\right)^{2}\right)\left(-1+\frac{1}{\Delta}\right) \\
& -\frac{(L-1)}{\Delta} \frac{\kappa(1+\kappa)}{\nu} \frac{N}{K} \frac{\left(\tilde{\delta}^{\star}\right)^{2}}{\left(1+\tilde{\delta}^{\star}\right)^{2}}\left(\frac{\alpha(1+\kappa)^{2}}{\nu}-2 \lambda \alpha^{2}(1+\kappa)^{2} \delta^{\star} \tilde{\delta}^{\star} \frac{N}{K}\right)
\end{aligned}
$$

Since $\frac{\widetilde{\delta}^{\star}}{\phi}=\frac{1}{N} \operatorname{tr}(\widetilde{\mathbf{T}})$ and $\widetilde{\mathbf{T}}$ is diagonal with equal entries, we have $[\widetilde{\mathbf{T}}]_{k, k}=\frac{N}{K} \frac{\widetilde{\delta}^{\star}}{\phi}$ such that (32) simplifies to

$$
F_{j}=F=\frac{\kappa}{1+\kappa} \frac{N}{K \phi} \frac{\left(\tilde{\delta}^{\star}\right)^{2}}{\left(1+\tilde{\delta}^{\star}\right)^{2}}
$$

and $\Delta_{j}$ in (33) reduces to 611 where $\vartheta=\vartheta_{j}=\frac{1}{N} \operatorname{tr}\left(\mathbf{T}_{j}^{2}\right)$. To further expand $\vartheta$, we use:

$$
\mathbf{T}_{j}^{2}\left(\lambda\left(1+\tilde{\delta}^{\star}\right) \mathbf{I}_{N}+\frac{1}{N} \frac{\overline{\mathbf{H}}_{j j} \overline{\mathbf{H}}_{j j}^{H}}{1+\delta^{\star} \phi}\right)=\mathbf{T}_{j}
$$

from which it follows that $\lambda\left(1+\tilde{\delta}^{\star}\right) \vartheta+\frac{1}{N^{2}} \frac{\operatorname{tr}\left(\overline{\mathbf{H}}_{j j}^{H} \mathbf{T}_{j}^{2} \overline{\mathbf{H}}_{j j}\right)}{1+\delta^{\star} \phi}=\delta^{\star}$ or equivalently:

$$
\begin{aligned}
\vartheta=\frac{\tilde{\delta}^{\star}}{\phi \lambda\left(1+\tilde{\delta}^{\star}\right)} & +\frac{1-\frac{K}{N}}{\lambda^{2}\left(1+\tilde{\delta}^{\star}\right)}-\frac{N}{K} \frac{\left(\tilde{\delta}^{\star}\right)^{2}}{\phi^{2}} \frac{\kappa}{(1+\kappa) \lambda\left(1+\tilde{\delta}^{\star}\right)^{2}} \\
& -\frac{1}{\lambda^{2}} \frac{1-\frac{K}{N}}{\left(1+\tilde{\delta}^{\star}\right)^{3}} \frac{N}{K} \frac{\left(\tilde{\delta}^{\star}\right)^{2}}{\phi} \frac{\kappa}{1+\kappa} .
\end{aligned}
$$

By using (60), we obtain

$$
\begin{aligned}
\vartheta & =\frac{-\frac{N}{K} \frac{\kappa}{(1+\kappa) \phi}\left(\lambda\left(\tilde{\delta}^{\star}\right)^{3}+\left(\tilde{\delta}^{\star}\right)^{2}\left(\lambda-\phi \frac{K}{N}+\phi\right)\right)}{\phi \lambda^{2}\left(1+\tilde{\delta}^{\star}\right)^{3}} \\
& +\frac{\tilde{\delta}^{\star} \phi-\frac{\kappa}{1+\kappa} \tilde{\delta}^{\star}+\phi}{\phi \lambda^{2}\left(1+\tilde{\delta}^{\star}\right)^{3}} .
\end{aligned}
$$

Putting all these results together, it follows that, if the simplified model in (53) is adopted, the SINR asymptotic approximations with RZF and S-MMSE reduce to

$$
\frac{1}{\frac{1}{N \rho^{\mathrm{dI}}} \frac{1}{\bar{\psi}} \frac{1}{\left(1-\lambda \frac{N}{K} \frac{\tilde{\delta}^{*}}{\phi}\right)^{2}}+\frac{\bar{s}}{\bar{\psi}\left(1-\lambda \frac{N}{K} \frac{\delta^{*}}{\phi}\right)^{2}}+\text { Coherent Interf. }}
$$

where we have used that $\phi_{j l k}=\frac{\alpha}{1+\kappa} \nu$ for $l \neq j$. Note that quantities $\bar{s}$ and $\bar{\psi}$ refers to $\bar{s}_{j k}$ and $\bar{\psi}_{j}$ used in Theorem 2 . It remains thus to compute $\bar{s}$ and $\bar{\psi}$ in the considered simplified model. We begin by treating $\bar{\psi}$. From (42), we obtain

$$
\begin{aligned}
\bar{\psi} & =\left(\lambda^{2} \frac{\vartheta}{\phi} \widetilde{\vartheta} \phi \frac{N}{K}+\frac{1-F}{\Delta \phi} \frac{N}{K} F\right)^{-1}=\left(-\frac{1}{\phi} \frac{N}{K}+\frac{1-F}{\Delta \phi} \frac{N}{K}\right)^{-1} \\
& =\frac{K \phi}{N}\left(-1+\frac{1}{\Delta}-\frac{\kappa}{1+\kappa} \frac{N}{K \phi} \frac{\left(\tilde{\delta}^{\star}\right)^{2}}{\left(1+\tilde{\delta}^{\star}\right)^{2}}\right)^{-1} \\
& =\frac{K \nu}{N(1+\kappa)^{2}}\left(-1+\frac{1}{\Delta}-\frac{\kappa N}{\nu K} \frac{\left(\tilde{\delta}^{\star}\right)^{2}(1+\kappa)}{\left(1+\tilde{\delta}^{\star}\right)^{2}}\right)^{-1}
\end{aligned}
$$

We now evaluate the interference term. Notice that $\xi_{j k}=\frac{1}{\psi}$ and $\zeta_{j k}=\frac{\lambda^{2} N}{K} \frac{\widetilde{\vartheta}}{\phi \Delta}$. Let $d=\frac{1}{1+\kappa}$. Hence, after standard calculus we obtain (118) from which (119) follows.

The computation of the coherent interference requires some preliminaries. From (60), we obtain

$\frac{\phi K}{N}\left(1+\tilde{\delta}^{\star}\right)^{2}=\lambda \tilde{\delta}^{\star}\left(1+\tilde{\delta}^{\star}\right)^{2}+\left(\tilde{\delta}^{\star}\right)^{2} \phi+\tilde{\delta}^{\star} \phi+\frac{\tilde{\delta}^{\star} \kappa}{1+\kappa}$

such that

$$
\frac{\phi K}{N \lambda}=\tilde{\delta}^{\star}+\frac{\tilde{\delta}^{\star}}{\lambda\left(1+\tilde{\delta}^{\star}\right)}\left(\phi+\frac{\frac{\kappa}{1+\kappa}}{\tilde{\delta}^{\star}+1}\right) .
$$

By using the identity $\frac{\phi K}{N \lambda}=\frac{\phi}{\lambda}+\tilde{\delta}^{\star}-\phi \delta^{\star}$ yields $\phi \delta^{\star}=$ $\frac{\phi}{\lambda\left(1+\tilde{\delta}^{\star}\right)}-\frac{\frac{\kappa}{1+\kappa} \tilde{\delta}^{\star}}{\lambda(1+\tilde{\delta})^{2}}$. By using these results, we get

$$
\begin{aligned}
& \sum_{l \neq j} \phi_{l j k}^{2} \delta^{2} \lambda_{l}^{2}\left[\widetilde{\mathbf{T}}_{l}\right]_{k k}^{2}\left(1-\lambda_{l}\left[\widetilde{\mathbf{T}}_{j}\right]_{k k}\right)^{-2}= \\
& =(L-1) \alpha^{2}\left(\delta^{\star}\right)^{2} \phi^{2}(1+\kappa)^{2}\left(\frac{1}{\lambda_{l}\left[\widetilde{\mathbf{T}}_{j}\right]_{k k}}-1\right)^{-2} \\
& =(L-1) \alpha^{2}(1+\kappa)^{2}\left(\frac{1}{\phi \delta^{\star}}-\frac{K}{N \lambda \tilde{\delta}^{\star} \delta^{\star}}\right)^{-2} \\
& =\left(\bar{L}-\frac{1}{1+\kappa}\right) \frac{\alpha}{\underline{\tau}^{2}} .
\end{aligned}
$$

\section{REFERENCES}

[1] T. L. Marzetta, "Noncooperative cellular wireless with unlimited numbers of base station antennas," IEEE Trans. Wireless Commun., vol. 9, no. 11 , pp. 3590-3600, 2010. 
[2] E. Larsson, O. Edfors, F. Tufvesson, and T. Marzetta, "Massive MIMO for next generation wireless systems," IEEE Commun. Mag., vol. 52, no. 2, pp. 186 - 195, February 2014.

[3] S. Parkvall, E. Dahlman, A. Furuskar, and M. Frenne, "Nr: The new 5G radio access technology," IEEE Commun. Std. Mag., vol. 1, no. 4, pp. 24-30, Dec 2017.

[4] T. L. Marzetta, E. G. Larsson, H. Yang, and H. Q. Ngo, Fundamentals of Massive MIMO. Cambridge University Press, 2016.

[5] E. Björnson, J. Hoydis, and L. Sanguinetti, "Massive MIMO has unlimited capacity," IEEE Trans. Wireless Commun., vol. 17, no. 1, pp. 574-590, Jan 2018.

[6] E. Bjornson, J. Hoydis, and L. Sanguinetti, "Massive MIMO networks: Spectral, energy, and hardware efficiency," Foundations and Trends in Signal Processing, vol. 11, no. 3-4, pp. 154-655, 2017. [Online]. Available: http://dx.doi.org/10.1561/2000000093

[7] J. Hoydis, S. Ten Brink, and M. Debbah, "Massive MIMO in the UL/DL of cellular networks: How many antennas do we need?" IEEE J. Sel. Areas Commun., vol. 31, no. 2, pp. 160-171, 2013.

[8] W. Hachem, M. Kharouf, J. Najim, and J. W. Silverstein, "A CLT for information-theoretic statistics of non-centered Gram random matrices," Random Matrices: Theory and Applications, vol. 1, no. 2, pp. 1-50, 2012. [Online]. Available: https://hal.archives-ouvertes.fr/hal-00829214

[9] W. Hachem, P. Loubaton, J. Najim, and P. Vallet, "On bilinear forms based on the resolvent of large random matrices," Annales de l'Institut Henri Poincar, Probabilities et Statistiques, vol. 49, no. 1, pp. $36-63$, 2013.

[10] E. Björnson, E. G. Larsson, and T. L. Marzetta, "Massive MIMO: ten myths and one critical question," IEEE Commun. Mag., vol. 54, no. 2, pp. 114-123, February 2016.

[11] L. Sanguinetti, A. Kammoun, and M. Debbah, "Asymptotic analysis of multicell massive MIMO over Rician fading channels," in 2017 IEEE International Conference on Acoustics, Speech and Signal Processing (ICASSP), March 2017, pp. 3539-3543.

[12] S. Wagner, R. Couillet, M. Debbah, and D. T. M. Slock, "Large system analysis of linear precoding in correlated MISO broadcast channels under limited feedback," IEEE Trans. Inf. Theory, vol. 58, no. 7, pp. 4509-4537, July 2012.

[13] J. Zhang, C. K. Wen, S. Jin, X. Gao, and K. K. Wong, "Large system analysis of cooperative multi-cell downlink transmission via regularized channel inversion with imperfect CSIT," IEEE Trans. Wireless Commun., vol. 12, no. 10, October 2013.

[14] L. Sanguinetti, R. Couillet, and M. Debbah, "Large system analysis of base station cooperation for power minimization," IEEE Trans. Wireless Commun., vol. 15, no. 8, pp. 5480-5496, Aug 2016.

[15] H. Falconet, L. Sanguinetti, A. Kammoun, and M. Debbah, "Asymptotic analysis of downlink MISO systems over rician fading channels," in Proceedings of the 41st IEEE International Conference on Acoustics, Speech and Signal Processing (ICASSP 2016), April 2016. [Online]. Available: http://arxiv.org/abs/1601.07024

[16] D. Yue and G. Y. Li, "LOS-based conjugate beamforming and powerscaling law in Massive MIMO systems," CoRR, vol. abs/1404.1654, 2014. [Online]. Available: http://arxiv.org/abs/1404.1654

[17] J. Hoydis, A. Kammoun, J. Najim, and M. Debbah, "Outage performance of cooperative small-cell systems under Rician fading channels," in IEEE 12th Int. Workshop Signal Processing Advances in Wireless Commun., June 2011, pp. 551-555.

[18] J. Zhang, C. K. Wen, S. Jin, X. Gao, and K. K. Wong, "On capacity of large-scale MIMO multiple access channels with distributed sets of correlated antennas," IEEE J. Sel. Areas in Commun., vol. 31, no. 2, pp. $133-148$, February 2013

[19] Q. Zhang, S. Jin, K. K. Wong, H. Zhu, and M. Matthaiou, "Power scaling of uplink massive MIMO systems with arbitrary-rank channel means," IEEE J. Sel. Topics Signal Process., vol. 8, no. 5, pp. 966-981, Oct 2014.

[20] H. AL-Salihi and F. Said, "Performance evaluation of Massive MIMO in correlated Rician and correlated Nakagami-m fading," in 2015 9th International Conference on Next Generation Mobile Applications, Services and Technologies, Sept 2015, pp. 222-227.

[21] Y. Huang, S. Ma, and Y. Wang, "Uplink achievable rate of fullduplex multi-cell Massive mimo systems," in 2015 IEEE International Conference on Communication Workshop (ICCW), June 2015, pp. 11311136.

[22] J. Zhang, L. Dai, X. Zhang, E. Björnson, and Z. Wang, "Achievable rate of Rician large-scale MIMO channels with transceiver hardware impairments," IEEE Trans. Veh. Technol., vol. 65, no. 10, pp. 88008806, Oct 2016.
[23] C. Masouros and M. Matthaiou, "Space-constrained Massive MIMO: Hitting the wall of favorable propagation," IEEE Commun. Lett., vol. 19, no. 5, May 2015.

[24] J. Zhang, C. Yuen, C. Wen, S. Jin, and X. Gao, "Ergodic secrecy sum-rate for multiuser downlink transmission via regularized channel inversion: Large system analysis," IEEE Commun. Lett., vol. 18, no. 9 , Sept 2014.

[25] J. Zhang, L. Dai, S. Sun, and Z. Wang, "On the spectral efficiency of Massive MIMO systems with low-resolution ADCs," IEEE Commun. Lett., vol. 20, no. 5, pp. 842-845, May 2016.

[26] J. Zhang, L. Dai, Z. He, S. Jin, and X. Li, "Performance analysis of mixed-ADC Massive MIMO systems over Rician fading channels," IEEE J. Sel. Areas Commun., vol. 35, no. 6, pp. 1327-1338, June 2017.

[27] Ö. Özdogan, E. Björnson, and E. G. Larsson, "Massive MIMO with spatially correlated rician fading channels," CoRR, vol. abs/1805.07972, 2018. [Online]. Available: http://arxiv.org/abs/1805.07972

[28] X. Gao, O. Edfors, F. Rusek, and F. Tufvesson, "Massive MIMO performance evaluation based on measured propagation data," IEEE Trans. Wireless Commun., vol. 14, no. 7, pp. 3899-3911, July 2015.

[29] I. Viering, H. Hofstetter, and W. Utschick, "Spatial long-term variations in urban, rural and indoor environments," in COST273 5th Meeting, Lisbon, Portugal, 2002.

[30] F. Rusek, D. Persson, B. K. Lau, E. Larsson, T. Marzetta, O. Edfors, and F. Tufvesson, "Scaling up MIMO: Opportunities and challenges with very large arrays," IEEE Signal Process. Mag., vol. 30, no. 1, pp. 40 60, Jan 2013

[31] S. M. Kay, Fundamentals of Statistical Signal Processing: Estimation Theory. Prentice Hall, 1993.

[32] H. Ngo, M. Matthaiou, and E. G. Larsson, "Performance analysis of large scale MU-MIMO with optimal linear receivers," in Proc. IEEE Swe-CTW, 2012, pp. 59-64.

[33] X. Li, E. Björnson, E. G. Larsson, S. Zhou, and J. Wang, "Massive MIMO with multi-cell MMSE processing: Exploiting all pilots for interference suppression," EURASIP J. Wireless Commun. Networking, 2017.

[34] E. Björnson, J. Hoydis, and L. Sanguinetti, "Pilot contamination is not a fundamental asymptotic limitation in Massive MIMO," in Proc. IEEE ICC, 2017.

[35] H. Q. Ngo, E. G. Larsson, and T. L. Marzetta, "Energy and spectral efficiency of very large multiuser MIMO systems," IEEE Trans. Commun., vol. 61, no. 4, pp. 1436-1449, 2013.

[36] J. Jose, A. Ashikhmin, T. L. Marzetta, and S. Vishwanath, "Pilot contamination and precoding in multi-cell TDD systems," IEEE Trans. Wireless Commun., vol. 10, no. 8, pp. 2640-2651, 2011.

[37] A. Kammoun, L. Sanguinetti, M. Debbah, and M.-S. Alouini, "Asymptotic analysis of RZF in large-scale MU-MIMO systems over Rician channels," submitted to IEEE Trans. Inf. Theory, 2018.

[38] H. Q. Ngo, E. G. Larsson, and T. L. Marzetta, "Aspects of favorable propagation in Massive MIMO," in Proceedings of the 22nd European Signal Processing Conference (EUSIPCO), Sept 2014, pp. 76-80.

[39] L. Sanguinetti, A. Kammoun, and M. Debbah, "Theoretical performance limits of Massive MIMO with uncorrelated Rician fading channels," Tech. Rep., 2018. [Online]. Available: http://www.iet.unipi.it/l.sanguinetti/techReportRician.pdf 


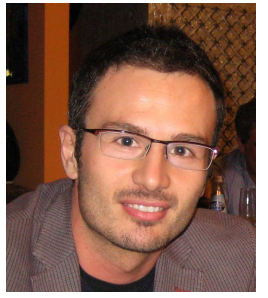

Luca Sanguinetti (SM'15) received the Laurea Telecommunications Engineer degree (cum laude) and the Ph.D. degree in information engineering from the University of Pisa, Italy, in 2002 and 2005 , respectively. Since 2005 he has been with the 'Dipartimento di Ingegneria dell'Informazione of the University of Pisa. In 2004, he was a visiting Ph.D. student at the German Aerospace Center (DLR), Oberpfaffenhofen, Germany. During the period June 2007 - June 2008, he was a postdoctoral associate in the Dept. Electrical Engineering at Princeton. During the period June 2010 - Sept. 2010, he was selected for a research assistantship at the Technische Universitat Munchen. From July 2013 to October 2017 he was with Large Systems and Networks Group (LANEAS), CentraleSupélec, Gif-sur-Yvette, France. He is currently an Associate Professor in the 'Dipartimento di Ingegneria dell'Informazione' of the University of Pisa, Italy.

Dr. Sanguinetti has co-authored the textbook Massive MIMO Networks: Spectral, Energy, and Hardware Efficiency (2017). He served as Exhibit Chair of the 2014 IEEE International Conference on Acoustics, Speech, and Signal Processing (ICASSP) and as the general co-chair of the 2016 Tyrrhenian Workshop on 5G\&Beyond. He served as an Associate Editor for IEEE TRANSACTIONS ON WIRELESS COMMUNICATIONS, and as Lead Guest Editor of IEEE JOURNAL ON SELECTED AREAS OF COMMUNICATIONS Special Issue on "Game Theory for Networks" and as an Associate Editor for IEEE JOURNAL ON SELECTED AREAS OF COMMUNICATIONS (series on Green Communications and Networking). Dr. Sanguinetti is currently serving as an Associate Editor for the IEEE SignAl PROCESSING LETTERS and is a member of the Executive Editorial Committee of IEEE TRANSACTIONS ON WIRELESS COMMUNICATIONS.

His expertise and general interests span the areas of communications and signal processing. He received the 2018 Marconi Prize Paper Award in Wireless Communications and co-authored a paper that received the young best paper award from the ComSoc/VTS Italy Section. He was the co-recipient of two best conference paper awards: IEEE WCNC 2013 and IEEE WCNC 2014 He was the recipient of the FP7 Marie Curie IEF 2013 "Dense deployments for green cellular networks".

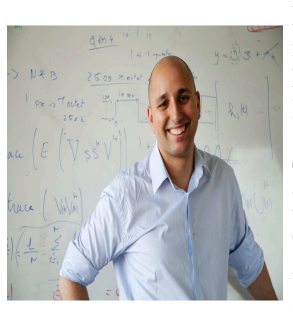

Mérouane Debbah (F15) entered the Ecole Normale Suprieure Paris-Saclay (France) in 1996 where he received his M.Sc and Ph.D. degrees respectively. He worked for Motorola Labs (Saclay, France) from 1999-2002 and the Vienna Research Center for Telecommunications (Vienna, Austria) until 2003. From 2003 to 2007, he joined the Mobile Communications department of the Institut Eurecom (Sophia Antipolis, France) as an Assistant Professor. Since 2007, he is a Full Professor at CentraleSupelec (Gifsur-Yvette, France). From 2007 to 2014, he was the director of the Alcatel-Lucent Chair on Flexible Radio. Since 2014, he is VicePresident of the Huawei France R\&D center and director of the Mathematical and Algorithmic Sciences Lab. His research interests lie in fundamental mathematics, algorithms, statistics, information \& communication sciences research.

$\mathrm{He}$ is an Associate Editor in Chief of the journal Random Matrix: Theory and Applications and was an associate and senior area editor for IEEE Transactions on Signal Processing respectively in 2011-2013 and 20132014. Mérouane Debbah is a recipient of the ERC grant MORE (Advanced Mathematical Tools for Complex Network Engineering). He is a IEEE Fellow, a WWRF Fellow and a Member émérite SEE. He has managed 8 EU projects and more than 24 national and international projects.

He received 19 best paper awards, among which the 2007 IEEE GLOBECOM best paper award, the Wi-Opt 2009 best paper award, the 2010 Newcom++ best paper award, the WUN CogCom Best Paper 2012 and 2013 Award, the 2014 WCNC best paper award, the 2015 ICC best paper award, the 2015 IEEE Communications Society Leonard G. Abraham Prize, the 2015 IEEE Communications Society Fred W. Ellersick Prize, the 2016 IEEE Communications Society Best Tutorial paper award, the 2016 European Wireless Best Paper Award, the 2017 Eurasip Best Paper Award and the 2018 IEEE Marconi Prize Paper Award as well as the Valuetools 2007, Valuetools 2008, CrownCom2009, Valuetools 2012, SAM 2014 and 2017 IEEE Sweden VT-COM-IT Joint Chapter Best Student paper awards. He is the recipient of the Mario Boella award in 2005, the IEEE Glavieux Prize Award in 2011 and the Qualcomm Innovation Prize Award in 2012.

\begin{abstract}
Abla Kammoun was born in Sfax, Tunisia. She received the engineering degree in signal and systems from the Tunisia Polytechnic School, La Marsa, and the Master's degree and the Ph.D. degree in digital communications from Telecom Paris Tech [then Ecole Nationale Superieure des Telecommunications (ENST)]. From June 2010 to April 2012, she has been a Postdoctoral Researcher in the TSI Department, Telecom Paris Tech. Then she has been at Supelec at the Alcatel-Lucent Chair on Flexible Radio until December 2013. Currently, she is working as a research scientist at KAUST university. Her research interests include performance analysis of wireless communication systems, random matrix theory, and statistical signal processing. She is the recipient of the SAM 2014 second Prize Best paper award.
\end{abstract}

